NBER WORKING PAPER SERIES

\title{
RISK REDUCTION IN LARGE PORTFOLIOS: WHY IMPOSING THE WRONG CONSTRAINTS HELPS
}

\author{
Ravi Jagannathan \\ Tongshu Ma \\ Working Paper 8922 \\ http://www.nber.org/papers/w8922 \\ NATIONAL BUREAU OF ECONOMIC RESEARCH \\ 1050 Massachusetts Avenue \\ Cambridge, MA 02138 \\ May 2002
}

We thank Torben Andersen, Gopal Basak, Louis Chan, Gregory Connor, Kent Daniel, Bernard Dumas, Ludger Hentschel, Philippe Henrotte, Ioulia Io .e, Andrew Lo, Jesper Lund, Robert McDonald, Steve Ross, Jay Shanken and seminar participants at Copenhagen Business School, Emory University, HEC, Insead, MIT, Norwegian School of Management, Hong Kong University of Science and Technology, University of Illinois at Urbana-Champaign, University of Iowa, University of Rochester, and University of Utah for helpful comments. We thank the editor, Richard Green, and two anonymous referees, for a number of suggestions that have helped us sharpen the focus of the article. We are responsible for any errors or omissions. The views expressed herein are those of the authors and not necessarily those of the National Bureau of Economic Research.

(C) 2002 by Ravi Jagannathan and Tongshu Ma. All rights reserved. Short sections of text, not to exceed two paragraphs, may be quoted without explicit permission provided that full credit, including (C) notice, is given to the source. 
Risk Reduction in Large Portfolios: Why Imposing the Wrong Constraints Helps

Ravi Jagannathan and Tongshu Ma

NBER Working Paper No. 8922

May 2002

JEL No. C49, G11, G12

\begin{abstract}
Mean-variance efficient portfolios constructed using sample moments often involve taking extreme long and short positions. Hence practitioners often impose portfolio weight constraints when constructing efficient portfolios. Green and Hollifield (1992) argue that the presence of a single dominant factor in the covariance matrix of returns is why we observe extreme positive and negative weights. If this were the case then imposing the weight constraint should hurt whereas the empirical evidence is often to the contrary. We reconcile this apparent contradiction. We show that constraining portfolio weights to be nonnegative is equivalent to using the sample covariance matrix after reducing its large elements and then form the optimal portfolio without any restrictions on portfolio weights. This shrinkage helps reduce the risk in estimated optimal portfolios even when they have negative weights in the population. Surprisingly, we also find that once the nonnegativity constraint is imposed, minimum variance portfolios constructed using the monthly sample covariance matrix perform as well as those constructed using covariance matrices estimated using factor models, shrinkage estimators, and daily data. When minimizing tracking error is the criterion, using daily data instead of monthly data helps. However, the sample covariance matrix without any correction for microstructure effects performs the best.
\end{abstract}

Ravi Jagannathan Department of Finance

Kellogg Graduate School of Management

Northwestern University

Evanston, IL 60208

and NBER

847-491-8338

rjaganna@nwu.edu
Tongshu Ma

Department of Finance

David Eccles School of Business

University of Utah

Salt Lake City, UT 84112

801-581-7506

fintsm@business.utah.edu 


\section{Introduction}

Markowitz's $(1952,1959)$ portfolio theory is one of the most important theoretical developments in finance. Mean-variance efficient portfolios play an important role in this theory. Such portfolios constructed using sample moments often involve taking large short positions in a number of assets and large long positions in some other assets. Since negative portfolio weights (short positions) are difficult to implement in practice, most investors impose the constraint that portfolio weights should be nonnegative when constructing mean-variance efficient portfolios.

Green and Hollifield (1992) argue that it is difficult to dismiss the observed extreme negative and positive weights as entirely due to imprecise estimation of the inputs used to construct mean-variance efficient portfolios. According to them the extreme weights in sample efficient portfolios are to a large extent due to the existence of a single dominance factor in the covariance structure of stock returns and the small amount of cross-sectional diversity in asset betas. To understand the Green and Hollifield argument, note that minimum variance portfolios can be constructed in two steps. First we naively diversify over the set of high beta stocks and the set of low beta stocks separately. The resulting two portfolios will have very low residual risk and different betas. Next we short the high beta portfolio and long the low beta portfolio to get rid of the systematic risk. Clearly, the second step involves taking extreme long and short positions when there is little dispersion in the betas of different assets. This is why minimum variance portfolios take large positive and negative positions in the underlying assets.

If extreme negative weights in efficient portfolios arise due to the presence of a single dominant factor then it would appear that imposing the nonnegativity constraints would lead to a loss in efficiency. However, empirical findings suggest that imposing these constraints improves the efficiency 
of optimal portfolios constructed using sample moments ${ }^{1}$. Does this mean that the evidence does not support the Green and Hollifield (1992) view?

In this paper we provide an answer to this question. We show that imposing the nonnegativity constraint on portfolio weights can help even when Green and Hollifield are right - i.e., the true covariance matrix is such that efficient portfolios involve taking large negative positions in a number of assets. We show that each of the no-shortsales constraints is equivalent to reducing the sample covariances of the corresponding asset with other assets by a certain amount. Stocks that have high covariances with other stocks tend to receive negative portfolio weights. Hence, to the extent that high estimated covariances are more likely to be caused by upward-biased estimation error, imposing the nonnegativity constraint can reduce the sampling error. It follows from the theory of shrinkage estimators that imposing the no-shortsales constraint can help even when the constraints do not hold in the population.

We also study the impact of upper bounds on portfolio weights since they are also commonly imposed by practitioners. We show that each upper bound constraint is equivalent to increasing the sample covariances of the corresponding asset with other assets by a certain amount. Since stocks that have low covariances with other stocks tend to get extreme high portfolio weights, and these extreme low estimated covariances are more likely to be caused by downward-biased estimation error, this adjustment in estimated covariances could reduce sampling error and help the out-of-sample performance of the optimal portfolios. Our empirical evidence suggests that imposing upperbounds on portfolio weights does not lead to a significant improvement in the out-of-sample performance of minimum risk portfolios when no-shortsales restrictions are already in place. Therefore the role of upperbounds is more to ensure that the minimum risk portfolios that come out of the optimization exercise can be constructed in practice.

\footnotetext{
${ }^{1}$ See Frost and Savarino (1988) for an excellent discussion.
} 
The most common estimator of a covariance matrix is the corresponding sample covariance matrix of historical returns. It has long been recognized that mean-variance efficient portfolios constructed using the sample covariance matrix perform poorly out-of-sample. ${ }^{2}$ The primary reason is that the large covariance matrices encountered in practice require estimating too many parameters. For example, the covariance matrix of the returns of 500 stocks has about 125,000 distinct parameters. However, monthly returns for all traded stocks are only available for the past 900 months or so. This gives less than 4 degrees of freedom per estimated parameter. Consequently the elements of the covariance matrix are estimated imprecisely.

Several solutions to this problem have been suggested. The first is to impose more structure on the covariance matrix to reduce the number of parameters that have to be estimated. This includes factor models and constant correlation models. The second approach is to use shrinkage estimators, shrinking the sample covariance matrix toward some target, such as the single-index model (Ledoit $(1996,1999))$. The third approach is to use data of higher frequency, for example, daily data in place of monthly data. These three approaches are widely used both by practitioners and academics.

For any covariance matrix estimator, we can impose the usual portfolio weight constraints. According to Green and Hollifield (1992), these constraints are likely to be wrong in population and hence they introduces specification error. According to our analysis, these constraints can reduce sampling error. Therefore the gain from imposing these constraints depends on the trade off between the reduction in sampling error and the increase in specification error. For covariance matrix estimators that have large sampling error, such as the sample covariance matrix, imposing these constraints is likely to be helpful. However, for the factor models and shrinkage estimators, imposing such constraint is likely to hurt. This is what we find empiri-

\footnotetext{
${ }^{2}$ See Jobson and Korkie (1980, 1981), Frost and Savarino (1986, 1988), Jorion (1986), Michaud (1989), Best and Grauer (1991), and Black and Litterman (1992).
} 
cally. Sampling errors are likely to be less important when the assets we use in constructing minimum variance portfolios are themselves large portfolios. In that case imposing nonnegativity constraints should hurt. Again this is what we find.

In this paper, we will focus on the effect of portfolio weight constraints on the global minimum variance portfolios. We do this because it has been reported in the literature that the global minimum variance portfolio has as large an out-of-sample Sharpe Ratio as other efficient portfolios. ${ }^{3}$ We also examine the effect of portfolio weight constraints on the performance of tangency portfolios and minimum tracking error portfolios. Our interest in minimum tracking error portfolios arises from the observation that it may be necessary in practice to construct portfolios using a subset of all available stocks that have low transactions costs and high liquidity to track certain benchmark indices that may contain assets that are not actively traded.

Our main empirical findings are the following:

1. For factor models and shrinkage estimators, imposing the usual portfolio weight constraint reduces the portfolio's efficiency slightly. On the

\footnotetext{
${ }^{3}$ Using monthly stock index return data for G7 countries Jorion (1985) convincingly argues that "... benefits from diversification are more likely to accrue from a reduction in risk." In the data set he examined, the global minimum variance portfolio had the best out of sample performance. It out performed classical tangent portfolio, the tangent portfolio construced using the Bayes-Stein estimator for the vector of mean returns, and the value weighted and equally weighted portfolios. Using simulation methods Jorion (1986) showed that this conclusion is robust if the sample size is not large. Jorion (1991) found that the minimum variance portfolio constructed using returns on seven industry stock index portfolios performed as well as the CRSP equally weighted and value weighted stock indices during the January 1926 to December 1987 period, in out of sample tests. The performance was comparable to that of the tangent portfolio constructed using the Bayes-Stein estimator for the mean. Bloomfield, Leftwich and Long (1977) found that portfolios constructed using mean-variance optimization did not dominate an equally weighted portfolio. On the other hand, Chan et al. (1999) documented that the constrained global minimum variance portfolios outperform the equally weighted portfolio.
} 
other hand, when the no-shortsales restriction is imposed, minimum variance and minimum tracking error portfolios constructed using the sample covariance matrix perform almost as well as those constructed using factor models, shrinkage estimators, or daily returns. ${ }^{4}$

2. When shortsales are allowed, minimum variance portfolios and minimum tracking error portfolios constructed using daily return sample covariance matrix perform the best. When covariance matrices are estimated using daily data, corrections for microstructure effects that have been suggested in the literature do not lead to superior performance. So far, this has not been recognized in the literature.

3. Tangency portfolios, whether constrained or not, do not perform as well as the global minimum variance portfolios in terms of out-of-sample Sharpe Ratio. This means that the estimates of the mean returns are so noisy that simply imposing the portfolio weight constraint is not enough, even though the constraints still have a shrinkage effect.

4. Monte Carlo simulations indicate that when nonnegativity constraints are in place, global minimum variance portfolios constructed using the sample covariance matrix can perform just as well as the covariance matrix estimators constructed by imposing the factor structure even when returns do have a dominant factor structure in the population.

5. In the simulations, imposing the nonnegativity constraint typically worsens the performance when a single factor model is used to estimate the covariance matrix. On the other hand, in the data, imposing nonnegativity constraints does not hurt much when using factor models. This suggests that stock returns probably do not have a stable time invariant factor structure.

\footnotetext{
${ }^{4}$ It is well recognized in the literature that imposing portfolio weight constraints leads to superior out-of-sample performance of mean-variance efficient portfolios. However to our knowledge no one has noticed that the performance improvement is so large that it is comparable to that attained using the other alternatives.
} 
The rest of the paper is organized as follows. In Section II, we provide a theoretical analysis of the shrinkage-like effect of imposing the no-shortsales restriction and upper bounds on portfolio weights when constructing global minimum risk portfolios. In Section III, we use simulation to evaluate the tradeoff between specification error and sampling error. This tradeoff clearly depends on the true covariance structure of the assets, which is not observed. We therefore calibrate the covariance structure in the simulation to that of the U.S. stocks. Using the simulation we provide some guidance as to when the nonnegativity constraint will start to hurt. In Section IV, we empirically examine the effects of portfolio weight constraints using the out of sample performances of the constrained and unconstrained optimal portfolios. In Section V, we discuss the role of portfolio weight constraints in constructing tangency portfolios. We conclude the paper in Section VI. Proofs and details about the construction of various covariance matrix estimates using daily returns are collected in the Appendices.

\section{The Effect of Portfolio Weight Constraints}

\section{A Some Theoretical Results}

Given an estimated covariance matrix $S$, the global portfolio variance minimization problem when portfolio weights are constrained to satisfy both a lower bound of zero and an an upper bound of $\bar{\omega}$ is given by:

$$
\begin{array}{ll}
\min _{\omega} & \omega^{\prime} S \omega \\
\text { s.t. } & \sum_{i} \omega_{i}=1 \\
& \omega_{i} \geq 0, \quad i=1,2, \cdots, N . \\
& \omega_{i} \leq \bar{\omega}, \quad i=1,2, \cdots, N .
\end{array}
$$


The Kuhn-Tucker conditions (necessary and sufficient) are

$$
\begin{aligned}
\sum_{j} S_{i, j} \omega_{j}-\lambda_{i}+\delta_{i}= & \lambda_{0} \geq 0, \quad i=1,2, \cdots, N . \\
\lambda_{i} \geq 0, & \text { and } \lambda_{i}=0 \text { if } \omega_{i}>0, i=1,2, \cdots, N . \\
\delta_{i} \geq 0, & \text { and } \delta_{i}=0 \text { if } \omega_{i}<\bar{\omega}, i=1,2, \cdots, N .
\end{aligned}
$$

Here $\lambda=\left(\lambda_{1}, \cdots, \lambda_{N}\right)^{\prime}$ are the Lagrange multipliers for the nonnegativity constraints $(3), \delta=\left(\delta_{1}, \cdots, \delta_{N}\right)^{\prime}$ the multipliers for the constraints (4), and $\lambda_{0}$ the multiplier for (2).

Denote a solution to the constrained portfolio variance minimization problem (1)-(4) as $\omega^{++}(S)$. Let 1 denote the column vector of ones. Then we have the following proposition.

\section{Proposition 1 Let}

$$
\tilde{S}=S+\left(\delta \mathbf{1}^{\prime}+\mathbf{1} \delta^{\prime}\right)-\left(\lambda \mathbf{1}^{\prime}+\mathbf{1} \lambda^{\prime}\right) .
$$

Then $\tilde{S}$ is symmetric and positive semi-definite, and $\omega^{++}(S)$ is one of its global minimum variance portfolios.

All proofs are given in Appendix B.

This result shows that constructing a constrained global minimum variance portfolio from $S$ is equivalent to constructing a (unconstrained) minimum variance portfolio from $\tilde{S}=S+\left(\delta \mathbf{1}^{\prime}+\mathbf{1} \delta^{\prime}\right)-\left(\lambda \mathbf{1}^{\prime}+\mathbf{1} \lambda^{\prime}\right)$. Later we will interpret $\tilde{S}$ as a shrunk version of $S$, and argue that this shrinkage can reduce sampling error.

In general, given a constrained optimal portfolio $\omega^{++}(S)$, there are many covariance matrix estimates that have $\omega^{++}(S)$ as their (unconstrained) minimum variance portfolio. Is there anything special about $\tilde{S}$ ? We do have an answer to this question when returns are jointly normal and $S$ is the MLE of the population covariance matrix. 
Let the $N \times 1$ return vector $h_{t}=\left(r_{1 t}, r_{2 t}, \cdots, r_{N t}\right)^{\prime}$ be iid normal $N(\mu, \Omega)$. Then the MLE of $\Omega$ is $S=\sum_{t=1}^{T}\left(h_{t}-\bar{h}\right)\left(h_{t}-\bar{h}\right)^{\prime} / T$. It is well known that for any estimate of the covariance matrix, the MLE of the mean is always the sample mean. (Morrison (1990), p.98.) With this estimate of the mean, the log-likelihood (as a function of the covariance matrix alone) becomes

$$
l(\Omega)=\mathrm{CONST}-\frac{T}{2} \ln |\Omega|-\frac{T}{2} \operatorname{tr}\left(S \Omega^{-1}\right) .
$$

This can also be considered as the likelihood function of $\Omega^{-1}$, and is defined for nonsingular $\Omega$.

Now consider the constrained MLE of $\Omega$, subject to the constraint that the global minimum variance portfolio constructed from $\Omega$ satisfy the weight constraints (3)-(4). Let $\Omega_{i, j}$ denote the $(i, j)$-th element of $\Omega$ and $\Omega^{i, j}$ denote the $(i, j)$-th element of $\Omega^{-1}$, then the constraints are

$$
\begin{aligned}
\sum_{j} \Omega^{i, j} & \geq 0, \quad i=1,2, \cdots, N . \\
\sum_{j} \Omega^{i, j} & \leq \bar{\omega} \sum_{k} \sum_{j} \Omega^{k, j} .
\end{aligned}
$$

So the constrained maximum likelihood (ML) problem is maximizing (9) subject to constraints (10)-(11). We have the following proposition.

Proposition 2 Assume that returns are jointly iid normal $N(\mu, \Omega)$. Let $S$ be the unconstrained MLE of $\Omega$.

1. Given $S$, let $\left\{\lambda_{i}, \delta_{i}, \omega_{i}\right\}_{i=1}^{N}$ be a solution to the constrained portfolio variance minimization problem (1) - (4), and construct $\tilde{S}$ according to (8). Assume $\tilde{S}$ is nonsingular. Then $\tilde{S}$ and $\left\{\lambda_{i},(1-\bar{\omega}) \delta_{i}\right\}_{i=1}^{N}$ jointly satisfy the first order conditions for the constrained $M L$ problem.

2. Let $\tilde{S}$ and $\left\{\lambda_{i}, \delta_{i}\right\}_{i=1}^{N}$ jointly satisfy the first order conditions for the constrained $M L$ problem. For $i=1, \cdots, N$, define $\omega_{i}=\sum_{j} \tilde{S}^{i, j} / \sum_{k} \sum_{l} \tilde{S}^{k, l}$, 
the normalized row sums of $\tilde{S}^{-1}$. Then $\left\{\lambda_{i}, \delta_{i} /(1-\bar{\omega}), \omega_{i}\right\}_{i=1}^{N}$ is a solution to the constrained portfolio variance minimization problem (1)(4), given $S$.

Roughly speaking, this proposition says that the $\tilde{S}$ constructed from the solution to the constrained global variance minimization problem is the ML estimator of the covariance matrix subject to the condition that the global minimum variance portfolio weights satisfy the nonnegativity and upper bound constraints. So we could impose the constraints in the estimation stage instead of the optimization stage and the result would be the same.

When only the nonnegativity constraint is imposed, the vector of Lagrange multipliers for the upper bound will be zero. So $\tilde{S}=S-\left(\lambda \mathbf{1}^{\prime}+\mathbf{1} \lambda^{\prime}\right)$, and we can simplify the statements in Proposition 2 in a straightforward way.

\section{B A Shrinkage Interpretation of the Effects of Portfo- lio Weight Constraints}

Let's first examine the effect of the nonnegativity constraint. Consider the unconstrained global portfolio variance minimization. The first order condition is

$$
\sum_{j=1}^{N} \omega_{j} S_{i, j}=\lambda_{0} \geq 0, \quad i=1,2, \cdots, N .
$$

The above condition says that at the optimum, stock $i$ 's marginal contribution to the portfolio variance is the same as stock $j$ 's, for any $i$ and $j$.

Suppose stock $i$ tends to have higher covariances with other stocks, i.e., the $i$ th row of $S$ tends to have larger elements than other rows. Then stock $i$ 's marginal contribution to the portfolio variance, $2 \sum_{j=1}^{N} \omega_{j} S_{i, j}$, will tend to be bigger than other stocks' marginal contributions. Therefore, to achieve optimality, we need to reduce stock $i$ 's portfolio weight. Stock $i$ may even have negative weight if its covariances with other stocks are sufficiently high. 
Therefore, a stock tends to receive a negative portfolio weight in the global minimum variance portfolio if it has higher variance and higher covariances with other stocks. In a one-factor structure, these are the high-beta stocks.

With only the nonnegativity constraint, Proposition 1 implies that constructing the constrained global minimum variance portfolio from $S$ is equivalent to constructing the (unconstrained) global minimum variance portfolio from $\tilde{S}=S-\left(\mathbf{1} \lambda^{\prime}+\lambda \mathbf{1}^{\prime}\right)$. Notice that the effect of imposing the constraint is that, whenever the nonnegativity constraint is binding for stock $i$, its covariances with other stocks, $S_{i j}, j \neq i$, are reduced by $\lambda_{i}+\lambda_{j}$, a positive quantity, and its variance is reduced by $2 \lambda_{i}$. We saw before that a stock will receive a negative portfolio weight in the minimum variance portfolio if its covariances are relatively too high. Therefore, the new covariance matrix estimate, $\tilde{S}$, is constructed by shrinking the large covariances toward the average covariances. Since the largest covariance estimates are more likely caused by upward-biased estimation error, this shrinking may reduce the estimation error.

We can interpret the effect of the upper bounds on portfolio weights similarly. In the unconstrained portfolio variance minimization, those stocks with low covariances with other stocks tend to receive high portfolio weights. So when the upper bound is imposed, it tends to be binding for these stocks. Proposition 1 says that constructing the upper-bound constrained minimum variance portfolio using $S$ is the same as constructing the unconstrained minimum variance portfolio from $\tilde{S}=S+\left(\mathbf{1} \delta^{\prime}+\delta \mathbf{1}^{\prime}\right)$. So the effect of imposing the constraint is that, whenever the upper bound is binding for stock $i$, its variance is raised by $2 \delta_{i}$ and its covariances with another stock $j$ is increased by $\left(\delta_{i}+\delta_{j}\right)$. Since the upper bound tends to be binding for those stocks with low covariances with other stocks, and low estimated covariances are more likely to be plagued by downward-biased estimation error, this adjustment may reduce sampling error and achieve better estimates of the covariance matrix in certain sense. This adjustment has a shrinkage-like effect. 
We now illustrate this shrinkage-like effect of portfolio weight constraints through an example. The covariance matrix estimate $S$ is the sample covariance matrix of a random sample of 30 stocks among all domestic common stocks that have all monthly returns from 1988 to 1997 (120 months). We consider the effect of imposing nonnegativity and an upper bound of $10 \%$ in the global portfolio variance minimization problem. Panel A of Table 1 reports summary statistics for the row sums of $S$, the portfolio weights of the unconstraind global minimum variance portfolio, and the Lagrangian multipliers for the nonnegativity constraint and the upper bound. We can see that both the row sums and the weights of the unconstrained optimal portfolio vary over a wide range: the lowest row sum is 0.0106, the highest is 0.0695; the lowest portfolio weight is -0.125 , and the highest is 0.357 .

Panel B of Table 1 reports the correlations among the row sums of $S$, the weights of the unconstrained portfolio, the Lagrange multipliers of the nonnegativity constraint, and the multipliers of the upper bound. We see that the correlation between the row sums and the unconstrained portfolio weights is -0.496 ; between the row sums and the multiplier of the nonnegativity constraint is 0.713 , and between the row sums and the multiplier of the upper bound is -0.425 . All of these correlations are significant at $2 \%$ level. Recall that the effect of the nonnegativity constraint is to revise $S$ into $S-\left(\lambda \mathbf{1}^{\prime}+\right.$ $\left.\mathbf{1} \lambda^{\prime}\right)$, and the additional upper bound is to further revise $S$ into $S-\left(\lambda \mathbf{1}^{\prime}+\right.$ $\left.\mathbf{1} \lambda^{\prime}\right)+\left(\delta \mathbf{1}^{\prime}+\mathbf{1} \delta^{\prime}\right)$, these correlations match well with our intuition discussed before. Specifically, those rows of $S$ that have low (or high) row sums tend to receive high (or low) weights in the unconstrained global minimum variance portfolio; the effect of the nonnegativity constraint tends to lower those rows with high row sums; and the effect of the upper bound tends to raise those rows with low row sums.

Table 1, Panel C and D report the effect of the weight constraints on the distinct individual covariances (i.e., $\left\{S_{i, j}, j \geq i\right\}$ ). Panel $\mathrm{C}$ gives the summary statistics of the distinct elements of $S$, the effect the nonnegativity 
constraint $\left(\lambda_{i}+\lambda_{j}\right)$ for $j \geq i$; and the effect of the upper bound constraint $\left(\delta_{i}+\delta_{j}\right)$ for $j \geq i$; Panel $\mathrm{D}$ reports the correlations among them.

Panel $\mathrm{C}$ shows that the individual covariances vary over a wide range: the lowest is -0.00186 and the highest is 0.0190 , while the mean covariance is 0.00139. Panel D shows that the correlation between $\left\{S_{i, j}, j \geq i\right\}$ and $\left\{\left(\lambda_{i}+\lambda_{j}\right), j \geq i,\right\}$ is 0.296 ; and the correlation between $\left\{S_{i, j}, j \geq i\right\}$ and $\left\{\left(\delta_{i}+\delta_{j}\right), j \geq i\right\}$ is -0.184 , and both are significant at $1 \%$ level. Again these correlations match our shrinkage-like interpretation of the effect of the portfolio weight constraint: the nonnegativity constraint tends to reduce the high covariances; and the upper bound tends to raise the low covariances.

Panel E shows the regression of $\left\{S_{i, j}, j \geq i\right\}$ on the adjustment due to the

portfolio weight constraints, $\left\{\lambda_{i}+\lambda_{j}, j \geq i\right\}$ and $\left\{\delta_{i}+\delta_{j}, j \geq i\right\}$, respectively. We see that the coeffcient estimates have the expected sign and are significant. Of course this is no surprise given the significant correlations reported in Panel D.

\section{A Simulation Study of the Tradeoff Be- tween Specification Error and Sampling Error}

In the last section, we argued that imposing the nonnegativity constraint in global portfolio variance minimization has a shrinkage-like effect. This shrinkage-like effect can reduce sampling error. On the other hand, the constraint is wrong in population when asset returns have a single dominant factor, as argued by Green and Hollifield (1992), because in that case the population global minimum variance portfolio takes extreme negative weights. Whether the nonnegativity constraint can help depends on the trade-off between these two effects: the reduction in sampling error on the one hand 
and the increase in the specification error on the other hand. That trade-off would depend on, among other things, the true covariance structure. Since we do not know the true covariance structure, we can not give a simple quantitative answer that holds true for all underlying covariance structures in the population. We therefore rely on using Monte Carlo simulation analysis in this section to further examine the trade-off between the two effects. By comparing the simulation results with the results using the real data, which are reported in the next section, we are also able to provide additional insights regarding the true covariance structure of the U.S. stock returns.

We assume a two-factor return structure in the simulation. ${ }^{5}$ We consider a two-factor structure because the literature often finds more than one factor in the return structure of the U.S. stocks. Without loss of generality, we set the factor variances to unity and the covariance between them to zero. We draw the betas for the first factor for each stock from an i.i.d Normal distribution with mean 1 and standard deviation $\sigma_{\beta}$, which varies from 0 to 0.4 across different sets of simulations. The stocks' betas with respect to the second factor are drawn from an i.i.d. Normal distribution with mean zero and standard deviation 0.2 . The residual variances are set to be constant over time, but cross-sectionally, they are drawn from an i.i.d. Lognormal $(0.8,0.7)$ distribution. When $\sigma_{\beta}=0.4$, the distribution of the betas with respect to the two factors roughly matches the empirical distribution of the first two betas for the NYSE stocks with the two factors being the first two of the five Connor-Korajczyk factors. Furthermore, the average fraction of variances that are residual variances also roughly matches the same fraction in the NYSE stocks.

We allow the number of stocks, $N$, to range from 30 to 300 . For each specification of $N$ and $\sigma_{\beta}$, we draw the betas and the residual variances for the stocks according to the afore mentioned distributional assumptions.

\footnotetext{
${ }^{5}$ We also performed a simulation with a one-factor structure. The results are qualitatively similar. These results are available upon request.
} 
These betas and residual variances are fixed for this specification even as we change the sample size $T$.

Given the sampled betas and residual variances, we then draw returns of different sample sizes. We first set $T=60$ and then vary it from $N+30$ to $N+210$ by increment of 60 . We allow $T=60$ because that is the sample size used in the simulation with real data in the next section, and having the same sample size allows us to compare the results.

Given the simulated returns data, we estimate the return covariance matrix, and form the nonnegativity constrained and unconstrained global minimum variance portfolios. We then calculate the ex post variances of these portfolios using the assumed true covariance matrix. This procedure is repeated ten times and the averages of ex post variances are reported.

We consider two covariance matrix estimators. The first is the sample covariance matrix. The second is the one-factor model. When the covariance matrix is estimated with the one-factor model, we assume the factor return is known, and is the return of the first factor used in the simulation.

Table 2 presents a subset of our simulation results. ${ }^{6}$ It examines the effects on imposing the nonnegativity constraint and/or the single-factor constraint. The table reports the percentage reductions in the ex post standard deviations of the global minimum variance portfolios constructed by imposing these constraints relative to that of the unconstrained global minimum variance portfolios constructed from the sample covariance matrix, and relative to the equal-weighted portfolio. The three panels, $\sigma_{\beta}=0, \sigma_{\beta}=0.2$, and $\sigma_{\beta}=0.4$, represents the cases where the nonnegativity constraint is correct, is severely wrong, and is moderately wrong, respectively. We report the cases where the number of stocks is either 30 or 300 , and the sample size is either 60 or 360 .

\footnotetext{
${ }^{6}$ To save space, we do not report the full result of the simulations, which is available upon request.
} 
Panel A shows that when the nonnegativity constraint is correct, imposing the nonnegativity constraint always helps. The gain from imposing the nonnegativity constraint is comparable to that obtained by imposing the one-factor structure. In fact, this is true for all the cases in our simulation, not only those cases reported in the table.

Across all three panels, the numbers in the last two columns are comparable. This means that if for some reason the nonnegativity constraint has to be imposed, then whether we use the sample covariance matrix or the one-factor model to estimate the covariance matrix makes little difference. Again this is true not only for the cases in Table 2 but for all the cases in our simulation.

When $\sigma_{\beta}>0$, so that the true global minimum variance portfolio has negative weights, the nonnegativity constraint always hurts if we use the one-factor model to estimate the covariance matrix, and the deteriation in performance is dramatic if the number of stocks is large. However, imposing the nonnegativity constraint helps when the sample covariance matrix is used and there are too many stocks relative to the length of the time series of observations used to estimate the covariance matrix. Again this is true in general and not only for the cases reported in the table.

Comparing the numbers on the last two columns in all three panels, we see that, paradoxically, the nonnegativity-constrained portfolios do worse than the equal-weighted index when the nonnegativity constraint is correct, and do better than the equal-weighted index when the nonnegativity constraint is wrong. This is because when there is dispersion in the betas (so the nonnegativity constraint is wrong in population), even if shortsales are not allowed, we can still reduce the exposure to systematic risk by investing only in the low-beta stocks. Thus we can achieve lower risk than the equalweighted index. On the other hand, when there is no dispersion in the betas (i.e., the nonnegativity constraint is correct in population), we can not reduce systematic risk at all, hence there is not much potential gain from 
optimal diversification. And the fact that we are using the estimated, not the true, covariance matrix can actually make things worse than a naive divsersification. In fact, throughout the simulation, we find that when $\sigma_{\beta}>$ $0,-$ i.e., when there is potential gain from optimal diversification, constrained optimal portfolios always do better than the equal-weighted index.

Based on the results from the full set of simulation, we also find a meaningful tradeoff between specification error and sampling error when $\sigma_{\beta}>0$ and the covariance matrix estimate is the sample covariance matrix. First, when $\sigma_{\beta}=0.1$, the nonnegativity constraint always helps. On the other hand, as $\sigma_{\beta}$ goes from 0.2 to 0.4 , we always find cutoff points for the sample size $T$, where when we have less observations than the cutoff points, the nonnegativity constraint helps, and when we have more observations than the cutoff points, the nonnegativity constraint hurts. These cutoff points increases as we increase the number of asset (as expected), but are not sensitive to changes in $\sigma_{\beta}$. These cutoff points are presented in Table 3. Recall that $\sigma_{\beta}=0.4$ roughly corresponds to the covariance structure of the NYSE stocks, and $\sigma_{\beta}=0.2$ or 0.3 represent cases where the nonnegativity constraint is more severely violated than when $\sigma_{\beta}=0.4$. Since these cutoff points are not sensitive to changes in $\sigma_{\beta}$ in this range, we can treat them as a rough guidline for practitioners who use U.S. stocks.

Table 4 gives the short interest in the unconstrained global miminum variance portfolios constructed from the true covariance matrix. This table gives us another metric about the extent to which the nonnegativity constraint is violated. 


\section{The Effect of Portfolio Weight Constraints: An Empirical Examination}

\section{A Data and Methodology}

In this section we examine empirically the effect of portfolio weight constraints. As we have said before, whether these weight constraints help or hurt is an empirical issue and will depend on the specific covariance matrix estimator. For the estimators that have large sampling errors such as the sample covariance matrix, the portfolio weight constraints are likely to be helpful, as documented by Frost and Savarino (1988) and demonstrated again in the simulation study in the last section. However, for the factor models and shrinkage estimators, the portfolio weight constraints are likely to be harmful.

We examine the effect of the portfolio weight constraints on the outof-sample performance of minimum variance and minimum tracking error portfolios formed using a number of covariance matrix estimators. This is done following the methodology in Chan, Karcesky, and Lakonishok (1999). At the end of each April from 1968 to 1998, we randomly choose 500 stocks from all common domestic stocks traded on the NYSE and the AMXE, with stock price greater than $\$ 5$, market capitalization more than the 80th percentile of the size distribution of NYSE firms, and with monthly return data for all the immediately preceding 60 months. We use return data for the preceding 60 months to estimate the covariance matrix of the returns of the 500 stocks. For estimators that use daily data, the daily returns during the previous 60 months of the same 500 stocks are used. When a daily return is missing, the equally-weighted market return of that day is used instead.

When variance minimization is the objective, we form three global minimum variance portfolios using each covariance matrix estimator - only two if 
the covariance matrix estimate is singular. The first portfolio is constructed without imposing any restrictions on portfolio weights, the second is subject to the constraint that portfolio weights should be nonnegative, and the third, in addition, faces the restriction that no more than 2 percent of the investment can be in any one stock. Each of these portfolios are held for one year, their monthly returns are recorded, and at the end of April of the next year, the same process is repeated. This gives at most three minimum variance portfolios that have post-formation monthly returns from May 1968 to April 1999 for each covariance matrix estimator. We use the standard deviation of the monthly returns on these portfolios to compare the different covariance matrix estimators.

For tracking error minimization, following Chan et al (1999) we assume the investor is trying to track the return of the S\&P 500 index. As in the case of portfolio variance minimization, we construct three tracking error minimizing portfolios for each covariance estimator. Notice that constructing the minimum tracking error variance portfolio is the same as constructing the minimum variance portfolio using returns in excess of the benchmark subject to the restriction that the portfolio weights sum to one.

\section{B Covariance Matrix Estimators}

The first estimator is the sample covariance matrix:

$$
S_{N}=\frac{1}{T-1} \sum_{t=1}^{T}\left(h_{t}-\bar{h}\right)\left(h_{t}-\bar{h}\right)^{\prime},
$$

where $T$ is the sample size, $h_{t}$ is a $N \times 1$ vector of stock returns in period $t$, and $\bar{h}$ is the average of these return vectors.

The second estimator assumes that returns are generated according to Sharpe's (1963) one-factor model given by:

$$
r_{i t}=\alpha_{i}+\beta_{i} r_{m t}+\epsilon_{i t},
$$


where $r_{m t}$ is the period $t$ return on the value-weighted portfolio of stocks traded on the NYSE, AMEX and NASDAQ. Then the covariance estimator is,

$$
S_{1}=s_{m}^{2} B B^{\prime}+D
$$

Here $B$ is the $N \times 1$ vector of $\beta$ 's, $s_{m}^{2}$ is the sample variance of $r_{m t}$, and $D$ has the sample variances of the residuals along the diagonal and zeros elsewhere.

The third estimator is the optimal shrinkage estimator of Ledoit (1999). It is a weighted average of the sample covariance matrix and the one-factor model-based estimator:

$$
S_{L}=\frac{\alpha}{T} S_{1}+\left(1-\frac{\alpha}{T}\right) S_{N},
$$

where $\alpha$ is a parameter that determines the shrinkage intensity that is estimated from the data. Ledoit (1999) shows this estimator outperforms the constant correlation model (Elton and Gruber (1973), Schwert and Seguin (1990)), the single-factor model, the industry factor model, and the principal component model with five principal components. ${ }^{7}$ For the fourth set of estimators we consider the Fama and French (1993) three-factor model, and the Connor and Korajczyk (1986, 1988) five-factor model and a three-factor version of it which includes only the first three of the five factors. This gives three additional covariance matrix estimators each corresponding to one of these multifactor models ${ }^{8}$.

Finally we consider several covariance matrix estimators that use daily return data. These include the daily return sample covariance matrix, daily one-factor model, daily Fama-French three-factor model, and daily ConnorKorajczyk five-factor and three-factor models. These models are similar to the corresponding monthly models. However, we incorporate the corrections

\footnotetext{
${ }^{7}$ For tracking error variance minimization, we also considered Ledoit's (1996) estimator that shrinks the sample covariance matrix toward the identity matrix. The results are similar and we do not report them.

${ }^{8}$ For tracking error variance minimization, the loadings on the first factor in these multifactor models are set to zero for every stock.
} 
for microstructure effects suggested by Scholes and Williams (1977), Dimson (1979) and Cohen et al. (1983). We also develop a new estimator for the covariance matrix of returns using daily return data that nests these three estimators. Details on the estimation of the monthly return covariance matrix using daily return data that allow for microstructure effects are provided in Appendix A.

\section{Empirical Results}

Table 5 gives the characteristics of minimum variance portfolios constructed using various covariance matrix estimates. ${ }^{9}$ Judging by the ex post standard deviations of the optimal portfolios, the shrinkage estimator proposed by Ledoit (1999) and the sample covariance matrix of daily return are the best performers. Since the Ledoit estimator is a particular weighted average of the one-factor model and the sample covariance matrix, we examined whether a simple average of the two estimators would do equally well. The random average of the one-factor model and the sample covariance matrix has an annualized out-of-sample standard deviation of 10.34 percentage points (not reported in Table 5) which is not much different from the 10.76 percentage points for the Ledoit estimator. We thus suspect that the sampling errors associated with the estimated optimal shrinkage intensity in the Ledoit estimator is rather large. Notice that even for these two best estimators, the unconstrained global minimum variance portfolios involing taking short positions that are over $80 \%$ of the portfolio value.

Next, we turn to the case when the no-shortsales restriction is imposed.

\footnotetext{
${ }^{9}$ To save space, we only report a subset of the simulation results in Tables 5-7. The results of using Connor-Korajczyk 3- and 5-factor models are very similar to those using the Fama-French 3-factor model. This is true when daily returns are used as well. So these results are not reported. Several versions of the daily one-factor model are dominated by the daily return sample covariance matrix. So these results are not reported either.
} 
There is a unique solution to the portfolio variance minimization problem using the sample covariance matrix although it is singular. Surprisingly the minimum variance portfolio constructed using the monthly sample covariance matrix compares favorably with all the other covariance matrix estimators. The out-of-sample annualized standard deviation is about 12 percentage points per year for all of the estimators, including the shrinkage estimator of Ledoit and the daily sample covariance matrix. Imposing the no-shortsales restriction leads to a small increase - between 8 and 14 percent - in the standard deviation of the minimum variance portfolios constructed using factor models. This decline in the performance is consistent with the observation by Green and Hollifield (1992) that shortsales restrictions probably do not hold in the population. The number of assets in the portfolio varies from a low of 24 for the sample covariance matrix estimator to a high of 65 for the daily sample covariance matrix. In comparison, the equally weighted portfolio of the 500 stocks has an annualized standard deviation of 17 percentage points, the value weighted portfolio of the 500 stocks has a standard deviation of 16 percentage points. A portfolio of randomly picked 25 stocks has a standard deviation of 18 percentage points which is 40 percent more than that of the optimal minimum variance portfolio constructed using the sample covariance matrix subject to the no-shortsales restrictions.

This clearly indicates that portfolio optimization can achieve a lower risk than naive diversification. Imposing an upper bound of two percentage points on portfolio weights in addition to a lower bound of zero does not affect the out-of-sample variance of the resulting minimum variance portfolios in any significant way.

When daily returns are used, the sample covariance matrix estimator performs the best when there are no portfolio weight constraints. The corrections for microstructure effects suggested in the literature do not lead to superior performance. When portfolio weights are constrained to be nonnegative all the models perform about equally well. 
Table 6 gives the corresponding numbers for the minimum tracking error portfolios. Several patterns emerge. Among the estimators that use monthly data, the monthly covariance matrix estimator and the one proposed by Ledoit (1996) perform the best. The former has only about 200 stocks whereas the latter has about 300 stocks.

The tracking error of the one factor model with non-negativity constraints is $5.04 \%$ which is rather large compared to the $3.36 \%$ for the sample covariance matrix estimator. This should not be surprising since the first dominant factor becomes less important for tracking error minimization. The portfolio weight constraints are not binding with the one factor model. This is strong support for the Green and Hollifield conjecture that large negative (and positive) weights are due to the presence of a single dominant factor which is effectively removed when we consider tracking error minimization.

The multifactor models of Connor and Korajczyk (not reported) as well as Fama and French perform better than the single factor model. This is consistent with the observations in Chan et al (1999). As is to be expected, portfolio weight constraints are not important when using multifactor models. However, the tracking error for the factor models is about one percentage point more than that for the sample covariance matrix and the Ledoit estimators.

With daily data we expect the precision of all the estimators to improve. If our conjecture that portfolio weight constriants lead to better performance due to the shrinkage effect then such constraints should become less important when daily data is used. This is what we find. When shortsales are allowed, the short interest is $36.6 \%$, a substantial amount. Imposing the nonnegativitiy constraint on portfolio weights reduces the number of stocks to 231. However, the performance is hardly affected. The tracking error goes down from $2.94 \%$ to $2.78 \%$.

With daily data, the sample covariance matrix estimator dominates all 
factor models, with the latter all perform equally well. Again corrections for microstructure effects make little difference in the ex post tracking error performance.

The value-weighted portfolio of the 500 stocks performs the best. This is to be expected since the benchmark is the S\&P500 portfolio, which is value weighted.

Table 7 reports the t-tests for the difference between the mean returns and mean squared returns of the portfolios compared with the nonnegativityconstrained portfolio from the monthly return sample covariance matrix. Since the differences in mean returns are all insignificant, the t-test for the difference in squared returns serve as a test for the difference in return variances, which is the focus of our study. We can see that for both portfolio variance minimization and tracking error minimization, once the nonnegativity constraint is imposed, the more sophisticated estimators do not in general give better out-of-sample performance than the monthly return sample covariance matrix. However, there is evidence that using daily returns can achieve smaller out-of-sample tracking error. Why using daily returns can help for the tracking error minimization case but not the total risk minimization case is an issue of future investigation.

\section{The Role of Constraints in Constructing Mean-Variance Efficient Portfolios}

In the previous sections, we mainly focused on the role of constraints on the global minimum variance portfolios. In this section, we will examine the same issue in the context of mean-variance efficient portfolios. For that purpose we will assume that there is a riskless asset. Then the mean variance efficient frontier is spanned by two assets: the riskless asset and the portfolio 
where the tangent from the risk free rate touches the mean variance frontier of asset returns. The global minimum variance portfolio will not be on the mean variance efficient frontier.

We will see that in this case, when the estimated covariance matrix is nonsingular, we can still give a shrinkage interpretation of the portfolio weight constraints, where the shrinkage is applied to the mean returns, not the covariance matrix. But we also point out that the sampling error in the estimated mean returns is too large for this shrinkage to be useful. Specifically, the constrained mean variance efficient portfolios perform worse out of sample than the global minimum variance portfolios, as have been reported by earlier studies. We will first present the theoretical analysis. Then we report some simulation results.

\section{A An Analysis of the Role of Portfolio Constraints on Mean-Variance Efficient Portfolios}

We use $w$ to denote the $N$ vector of portfolio weights on the risky assets; $S$ the covariance matrix of the excess returns (over the risk free rate); and $\mu$ the vector of excess returns.

Suppose we want to find the minimum variance portfolio with expected excess return $k$. Let's first consider the case where there is no constraints on individual portfolio weights. The Lagrangian is

$$
L=\frac{1}{2} w^{\prime} S w-\theta\left(w^{\prime} \mu-k\right),
$$

where $\theta$ is the scalar Lagrange multiplier. The first order conditions are given by

$$
\begin{aligned}
S w-\theta \mu & =0, \\
w^{\prime} \mu & =k .
\end{aligned}
$$


From this we can find the optimal portfolio weights. These are standard results.

Now let's consider the constrained efficient portfolio problem, where the portfolio weights are subject to known lower and upper bounds: ${ }^{10}$

$$
0 \leq w_{i} \leq \bar{w}_{i}
$$

The Lagrangian is

$$
L=\frac{1}{2} w^{\prime} S w-\theta\left(w^{\prime} \mu-k\right)-\lambda^{\prime} w+\delta^{\prime}(w-\bar{w}),
$$

where $\lambda$ and $\delta$ are vectors of nonnegative Lagrange multipliers.

The first order conditions are

$$
\begin{aligned}
S w-\theta \mu-\lambda+\delta & =0, \\
\lambda_{i} w_{i} & =0, \\
\delta_{i}\left(w_{i}-\bar{w}_{i}\right) & =0, \\
w^{\prime} \mu-k & =0 .
\end{aligned}
$$

Suppose for a given $k$, the solution to the constrained minimum variance portfolio problem is $\left(w^{*}(k), \theta(k), \lambda(k)\right)$. Assume that in a neighborhood of $k$, the set of binding lower and upper bounds stays the same. We know that the set of binding lower and upper bounds only changes at a finite number of points as $k$ varies. Since in practice $S$ and $\mu$ are estimated and hence are random, so with probability one, the above assumption is true. Now in this neighborhood of $k$, we can re-write the binding lower and upper bounds as equality constraints. So locally we are dealing with a constrained

\footnotetext{
${ }^{10} \mathrm{Liu}(2001)$ examined the same problem, though our approach here is different from his. He works with the utility maximization problem of a mean-variance utility investor. We work on the variance minimization for a given target return. We also point out that the shrinkage interpretation is only valid when $S$ is nonsingular.
} 
optimization with only equality constraints. Further, both the solution to this constrained optimization problem and the mimimum portfolio variance change smoothly with respect to $k$. Applying the envelope theorem, we have

$$
\frac{\partial\left(\frac{1}{2} w^{* \prime} S w^{*}\right)}{\partial k}=-\theta \frac{\partial\left(w^{\prime} \mu-k\right)}{\partial k}=\theta
$$

The envelope theorem simplifies here because neither the objective function nor the equality constraints depend on $k$ directly. Since on the efficient frontier there will be a positive trade-off between portfolio variance and portfolio expected return, the LHS of the above equation, and hence $\theta$, is positive.

Since $\theta$ is positive, we can re-write the first order condition (15) as

$$
S w-\theta\left(\mu+\frac{1}{\theta} \lambda-\frac{1}{\theta} \delta\right)=0
$$

Notice this is in the same form of the first order condition for the unconstrained portfolio variance minimization problem (14). Therefore, constructing the constrained portfolio variance minimizaition from $(S, \mu)$ with target expected excess return $k$ is the same as constructing the unconstrained one from $\left(S, \mu+\frac{1}{\theta} \lambda-\frac{1}{\theta} \delta\right)$ with target expected excess return $\left(k-\frac{1}{\theta} \delta^{\prime} \bar{w}\right)$, because the constraint on the portfolio mean return is now:

$$
w^{\prime}\left(\mu+\frac{1}{\theta} \lambda-\frac{1}{\theta} \delta\right)=k-\frac{1}{\theta} \delta^{\prime} \bar{w}
$$

due to (16) - (18). (Notice that if only the nonnegativity constraint is present, then the target return stays the same since $\delta$ is the zero vector.)

We point out that the above equivalence is true only when $S$ is nonsingular. When $S$ is singular, such as the monthly return sample covariance matrix estimator in our empirical work in the earlier section, the unconstrained mean-variance optimization has no solution, but the constrained one still has a solution since the domain of the objective function is now compact. So we don't have the equivalence result anymore. The fact that the constrained minimum variance portfolio can be constructed even though 
$S$ is singular implies that the constraint implies some adjustment to $S$. Liu (2001) ignored this point.

Assume $S$ is nonsingular, then if the nonnegativity constraint is binding for stock $i$, its mean return is adjusted upward by the amount $\frac{1}{\theta} \lambda_{i}$. If the upper bound is binding for stock $j$, its mean return is adjusted downward by the amount $\frac{1}{\theta} \delta_{j}$. In the unconstrained minimum variance portfolio, stocks with low expected returns tends to get negative weights, so the nonnegativity constraint will be binding. Imposing the nonnegativity constraint is as if we adjust their expected returns upwards. The upper bounds have similar effects. So imposing the weight constraints is equivalent to shrinking the mean estimates.

\section{B Some Empirical Results}

In this subsection, we empirically examine the shrinkage effect of imposing portfolio weight constraints in the mean-variance optimization problem. We will compare the out-of-sample performances of the constrained tangency portfolios with that of the unconstrained ones, and with that of the global minimum variance portfolios. We use the historical average return on an asset as the measure of expected return on that asset. This would be a more reasonable assumption for large stock portfolios than for individual stocks. We therefore work with assets that are themselves portfolios of many stocks. In particular, we construct these optimal portfolios using the Fama-French 25 size/book-to-market sorted stock portfolios.

At the end of April each year from 1968 to 1998, we use monthly returns of the past 5 years to estimate the mean returns and return covariance matrix of the 25 assets. We use several covariance matrix estimators, including the sample covariance matrix, the one-factor model (where the factor return is the value-weighted market index), the Fama-French three factor model, and the Ledoit estimator. Using the estimates of mean returns and return co- 
variance matrices, we form the unconstrained and nonnegativity constrained tangency portfolios and the global minimum variance portfolios. We hold these portfolios for one year, and then repeat the procedure again. The realized returns of these optimal portfolios are recorded and examined.

The results are presented in Table 8. Panel A gives the out-of-sample performance of the tangency portfolios. Panel B gives the corresponding numbers for the global minimum variance portfolios. We use the Sharpe Ratio to compare the performances of different portfolios. We draw the following conclusions from Table 8: (1) The constrained tangency portfolios fared better than the unconstrained ones. This means there is some beneficial shrinkage associated with imposing the constraints. (2) The constrained tangency portfolios fared worse than the constrained global minimum variance portfolios. This means that the sampling error in the estimated mean returns is still too large even after the shrinking induced by imposing the constraints. Hence there is a need to bring in other information about expected returns. This justifies our focus on the global minimum variance portfolios. (3) The constrained global minimum variance portfolios did worse than the corresponding unconstrained ones. This is true even if we use the sample covariance matrix. This contrasts sharply with the simulation result in Table 2, where for the case of 30 stocks, 60 months observations, and the covariance matrix is estimated using the sample covariance matrix, the constraint always helps. Recall that the simulation there was calibrated to the covariance structure of individual stocks. The covariance matrix of returns on a collection of assets that are themselves portfolios of a large collection of stocks can be measured with substantially more precision than the covariance matrix of returns on individual stocks. Hence when we are working with assets that are themselves porfolios of large number of stocks, imposing the nonnegativity constraint hurts. This provides strong support for Green and Hollifield (1992). Also, the fact that the constraint helps there but not here presents a clear example that the gain from imposing the constraint depends on the amount of sampling error. 


\section{Concluding Remarks}

Investors often voluntarily impose no-shortsales restrictions in optimal risk reduction. However, Green and Hollifield (1992) argue that the optimal portfolios may well involve extreme positive and negative weights. We reconcile this apparent contradiction. We show that imposing the no-shortsales constraint has a shrinkage-like effect. It follows from the theory of shrinkage estimators that imposing the constraint can help even when the constraint does not hold in the population. The upper bounds on portfolio weights have similar shrinking effects. Using a Monte Carlo simulation we show that this is indeed what is happening in optimal portfolio risk reduction.

Imposing the weight constraints also introduces specification error, since these constraints are violated in population, as argued by Green and Hollifield. So the net effect depends on the tradeoff between the reduction in sampling error and the increase in specification error, and whether the net effect is positive or negative is an empirical issue. Frost and Savarino (1988) and our simulation study show that for the sample covariance matrix, imposing the weight constraints helps. Our empirical results also show that for factor models and shrinkage estimators, imposing the weight constraints hurts slightly. On the other hand, once the no-shortsales restriction is imposed, portfolios constructed from the sample covariance matrix perform as well as portfolios constructed using covariance matrices estimated using factor models and shrinkage methods.

While the effect of the portfolio weight constraints has been examined empirically in the literature before, our result on the shrinkage effect of such constraints is the first "theoretical" result on this issue. One potential use of this result is to gauge the tightness of a set of portfolio weight constraints by the change from $S$ to $\tilde{S}$, as measured by $\|S-\tilde{S}\|$ for some matrix norm $\|\cdot\|$. A large $\|S-\tilde{S}\|$ means that the effect of the constraints is quite strong and the constraints are quite tight. This measure of tightness can help portfolio 
managers to decide the appropriate lower and upper bounds on portfolio weights when constructing the optimal portfolios.

Our empirical findings raise the following questions that deserve further examination. (a) Why the use of daily data can help achieve better tracking error reduction but not portfolio variance reduction? A possible explanation is that covariances caused by exposure to the dominant factor change much more over time when compared to those that are caused by exposure to other factors. While the use of daily return data helps to reduce the sampling error it does not take into account time variations in the covariance structure. This may be less important once the dominant factor is removed as in the case of tracking error minimization. (b) Why corrections for microstructure effects do not help even though they provide more precise estimates of factor betas? This could be due to the greater instability of microstructure effects across stocks and over time. (c) Why do we find large in-sample-optimism even for factor models and shrinkage estimators? Past researchers appear to attribute this to sampling error. Additional Monte Carlo simulations indicate that when returns have an exact factor structure, and the covariance matrices are constructed using estimated factors, the in-sample optimism is attenuated. This means that sampling error alone is unlikely to provide a complete answer. Part of the reason has to be that either returns do not have an exact factor structure or that the covariance structure is changing over time. Hence shrinkage methods and factor models, which are intended to reduce sampling errors, can only achieve limited success.

\section{Appendix A: Covariance Matrix Estima- tors that Use Daily Returns}

This appendix describes how we estimate monthly covariance matrices us-

ing daily return data after taking into account serial correlations and cross- 
correlations at various leads and lags induced by microstructure effects. We consider the estimators proposed by Scholes and Williams (1977), Dimson (1979), and Cohen, et al (1983, hereafter CHMSW). We point out that the CHMSW estimator of the sample covariance matrix is usually not positive semi-definite. We then propose a new methodology to estimate the covariance matrices of monthly returns using data on daily returns that are always positive semi-definite. Our estimator uses the fact that the continuously componded monthly return is the sum of continuously compounded daily returns. Our estimator can be readily modified based on restrictions imposed by microstructure models.

The CHMSW estimator is based on the following relationship:

$$
\begin{aligned}
\operatorname{cov}\left(r_{j, t}^{t}, r_{k, t}^{t}\right)= & \operatorname{cov}\left(r_{j, t}, r_{k, t}\right)+\sum_{n=1}^{L} \operatorname{cov}\left(r_{j, t}, r_{k, t-n}\right) \\
& +\sum_{n=1}^{L} \operatorname{cov}\left(r_{j, t-n}, r_{k, t}\right)
\end{aligned}
$$

for any pair of stocks $j$ and $k, j \neq k$. Here $r^{t}$ denotes the true date $t$ return and $r$ denotes the observed return, $t$ and $t-n$ denote dates $t$ and $t-n$. Based on this relation we can estimate $\operatorname{cov}\left(r_{j, t}^{t}, r_{k, t}^{t}\right)$ using observed daily returns. In practice, $L$ is set to three and the variances are estimated using sample variances without any adjustment (Cohen et al. (1983) and Shanken (1987)). The Scholes-Williams estimator is the special case of the above, with $L$ set to one. The estimate of the full sample covariance matrix using the CHMSW method is denoted as "Daily Sample Covariance Matrix (CHMSW)" in Tables 4-6.

Equation (19) is also valid if either asset (or both) is a portfolio. For a well-diversified portfolio, (19) is approximately valid for its variance also. Based on this, we can estimate a stock's beta as its covariance with the market portfolio divided by the market portfolio's variance. The daily onefactor models (Scholes-Williams and CHMSW) in Tables 4-6 are estimated using this strategy. 
There is a problem with these estimators. The estimated covariance matrix, constructed from individual covariances and variances, is not positive semi-definite. This is problematic for portfolio optimization. We propose a new estimator that does not have this problem. ${ }^{11}$ Notice that monthly log returns are simply sums of daily log returns:

$$
r_{i, \tau}=\sum_{t=(\tau-1) m+1}^{\tau m} r_{i, t} .
$$

Here $\tau$ is month $\tau, t$ is day $t$, and $m$ is the number of days in a month. Then the monthly return covariance is

$$
\operatorname{cov}\left(r_{i, \tau}, r_{j, \tau}\right)=\sum_{t=(\tau-1) m+1}^{\tau m} \sum_{s=(\tau-1) m+1}^{\tau m} \operatorname{cov}\left(r_{i, t}, r_{j, s}\right) .
$$

Unlike the CHMSW estimator (19), the above is valid for any $i$ and $j$, even if either one (or both) is a portfolio. Assuming covariance stationarity as usual, we can drop the time subscripts, and get

$$
\begin{aligned}
\operatorname{cov}^{M}\left(r_{i}, r_{j}\right) & =m \cdot \operatorname{cov}^{D}\left(r_{i, t}, r_{j, t}\right) \\
& +(m-1) \cdot\left(\operatorname{cov}^{D}\left(r_{i}, r_{j, t+1}\right)+\operatorname{cov}^{D}\left(r_{i, t+1}, r_{j, t}\right)\right) \\
& +(m-2) \cdot\left(\operatorname{cov}^{D}\left(r_{i}, r_{j, t+2}\right)+\operatorname{cov}^{D}\left(r_{i, t+2}, r_{j, t}\right)\right) \\
& +\cdots \\
& +\left(\operatorname{cov}^{D}\left(r_{i, t}, r_{j, t+m-1}\right)+\operatorname{cov}^{D}\left(r_{i, t+m-1}, r_{j, t}\right)\right) .
\end{aligned}
$$

The superscripts $M$ and $D$ denote the covariance of the monthly returns and the covariance of the daily returns, respectively. We set $m=21$ for there are about 21 trading days in one month.

An obvious approach is to use the sample counterpart of the RHS of (20) to estimate the monthly return covariance. However, to guarantee that

\footnotetext{
${ }^{11}$ There is a difference between our approach and the approach taken by Scholes and Williams and CHMSW. While they want to estimate the "true" covariances and betas using the daily returns, we want to use daily returns to estimate the covariances and betas of monthly returns.
} 
the covariance matrix is positive semi-definite, we need to further adjust the covariance estimates (20) slightly. Let

$$
R_{0}=\left(r_{t, i}-\overline{r_{., i}}\right)_{t=1, \ldots, T ; i=1, \ldots, N}
$$

the matrix of demeaned returns. For $j=1, \cdots, m-1$, let $R_{-j}$ be the same size as $R_{0}$, with the first $j$ rows set to zeros, and the remaining $T-j$ rows the same as $R_{0}$ 's first $T-j$ rows (i.e., $R_{-j}$ is the matrix of lag- $j$ demeaned returns). Let

$$
\begin{gathered}
\Omega_{j}=R_{0}^{\prime} R_{-j} / T \\
S=m \Omega_{0}+\sum_{j=1}^{m-1}(m-j)\left(\Omega_{j}+\Omega_{j}^{\prime}\right) .
\end{gathered}
$$

Then $S$ is positive semi-definite (see Newy and West (1987) for the proof) and $S$ is a consistent estimator of the RHS of (20). The covariance matrix estimated this way is denoted as "Daily Sample Covariance Matrix (New)" in Tables $5-7$.

If we assume a $k$-factor model, then the beta estimates are

$$
b=(\operatorname{vâr}(f))^{-1} \operatorname{côv}(f, r) \text {. }
$$

Here $b$ is $k \times N$, vâr $(f)$ is the estimated factor covariance matrix (of size $k \times k)$ and is estimated according to $(21)$, and $\operatorname{cov}(f, r)$ is estimated (similar to $(21))$ by

$$
\operatorname{covv}(f, r)=\frac{1}{T}\left[m F_{0}^{\prime} R_{0}+\sum_{j=1}^{m-1}(m-j)\left(F_{0}^{\prime} R_{-j}+F_{-j}^{\prime} R_{0}\right)\right],
$$

with $F_{-j}$ defined similarly as $R_{-j}$.

For factor models, the residual covariance matrix is assumed diagonal and the residual variances are estimated by the sample variances of residuals calculated from observed stock returns, observed factor returns, and the estimated betas. In Tables 5-7, the estimators denoted as "Daily 1 Factor 
Model (New)," "Daily Connor-Korajczyk 3 Factor Model," "Daily ConnorKorajczyk 5 Factor Model," and "Daily Fama-French 3 Factor Model," are all estimated using this strategy.

We construct the daily Connor-Korajczyk factors and Fama-French factors by following the same procedure as outlined in Connor and Korajczyk (1988) (using daily returns instead of monthly returns) and Fama and French (1993).

Finally, in Tables 5-7, the estimator "Daily Sample Covariance Matrix" denotes the sample covariance of daily returns.

\section{Appendix B: Proof of the Propositions}

Proof of Proposition 1: ${ }^{12} \tilde{S}$ is obviously symmetric. Now we prove that it is positive demi-definite. Suppose that

$$
\left(\omega_{1}, \cdots, \omega_{N}, \lambda_{1}, \cdots, \lambda_{N}, \delta_{1}, \cdots, \delta_{N}, \lambda_{0}\right) \equiv\left(\omega^{\prime}, \lambda^{\prime}, \delta^{\prime}, \lambda_{0}\right)
$$

is a solution to the constrained portfolio variance minimization problem (1) - (4). For any vector $x$,

$$
x^{\prime} \tilde{S} x=x^{\prime} S x-x^{\prime}\left(\mathbf{1} \lambda^{\prime}+\lambda \mathbf{1}^{\prime}\right) x+x^{\prime}\left(\mathbf{1} \delta^{\prime}+\delta \mathbf{1}^{\prime}\right) x=x^{\prime} S x-2\left(x^{\prime} \mathbf{1}\right)\left(x^{\prime}(\lambda-\delta)\right) .
$$

(Here $\mathbf{1}$ is a vector of ones.) By the first order condition, $\lambda-\delta=S \omega-\lambda_{0} \mathbf{1}$. Hence, $x^{\prime}(\lambda-\delta)=x^{\prime} S \omega-\lambda_{0} x^{\prime} \mathbf{1}$. Therefore

$$
2\left(x^{\prime} \mathbf{1}\right)\left(x^{\prime}(\lambda-\delta)\right)=2\left(x^{\prime} \mathbf{1}\right)\left(x^{\prime} S \omega\right)-2 \lambda_{0}\left(x^{\prime} \mathbf{1}\right)^{2} .
$$

\footnotetext{
${ }^{12}$ We thank Gopal Basak for providing a part of this proof.
} 
But

$$
\left|\left(x^{\prime} \mathbf{1}\right)\left(x^{\prime} S \omega\right)\right|=\left|\left(x^{\prime} \mathbf{1}\right)\left(x^{\prime} S^{1 / 2}\right)\left(S^{1 / 2} \omega\right)\right| \leq\left|\left(x^{\prime} \mathbf{1}\right)\right|\left(x^{\prime} S x\right)^{1 / 2}\left(\omega^{\prime} S \omega\right)^{1 / 2} .
$$

The first equality holds since $S$ is positive semi-definite, and the last inequality is due to the Cauchy-Schwarz inequality.

Again from the First Order Condition,

$$
0 \leq \omega^{\prime} S \omega=\omega^{\prime} \lambda-\omega^{\prime} \delta+\lambda_{0} \omega^{\prime} \mathbf{1}=\lambda_{0}-\bar{\omega} \delta^{\prime} \mathbf{1} \leq \lambda_{0}
$$

So

$$
\left|\left(x^{\prime} \mathbf{1}\right)\left(x^{\prime} S \omega\right)\right| \leq\left|\left(x^{\prime} \mathbf{1}\right)\right|\left(x^{\prime} S x\right)^{1 / 2}\left(\lambda_{0}\right)^{1 / 2}
$$

Combining the above inequality with (22) and (23), we have

$$
\begin{aligned}
x^{\prime} \tilde{S} x & =x^{\prime} S x-2\left(x^{\prime} \mathbf{1}\right) x^{\prime} S \omega+2 \lambda_{0}\left(x^{\prime} \mathbf{1}\right)^{2} \\
& \geq x^{\prime} S x-2\left|\left(x^{\prime} \mathbf{1}\right) x^{\prime} S \omega\right|+2 \lambda_{0}\left(x^{\prime} \mathbf{1}\right)^{2} \\
& \geq x^{\prime} S x-2\left|\left(x^{\prime} \mathbf{1}\right)\right|\left(x^{\prime} S x\right)^{1 / 2}\left(\lambda_{0}\right)^{1 / 2}+2 \lambda_{0}\left(x^{\prime} \mathbf{1}\right)^{2} \\
& =(a-b)^{2}+b^{2}
\end{aligned}
$$

where $a=\left(x^{\prime} S x\right)^{1 / 2}$ and $b=\lambda_{0}^{1 / 2}\left|\left(x^{\prime} \mathbf{1}\right)\right|$. Obviously this is always nonnegative. So $\tilde{S}$ is positive semi-definite.

Because $\tilde{S}$ is positive semi-definite, to show that $\omega=\left(\omega_{1}, \cdots, \omega_{N}\right)$ is a (unconstrained) global minimum variance portfolio of $\tilde{S}$, it suffices to verify the first order condition:

$$
\begin{aligned}
\tilde{S} \omega & =S \omega-\left(\mathbf{1} \lambda^{\prime}+\lambda \mathbf{1}^{\prime}\right) \omega+\left(\mathbf{1} \delta^{\prime}+\delta \mathbf{1}^{\prime}\right) \omega \\
& =S \omega-\lambda \mathbf{1}^{\prime} \omega+\mathbf{1} \bar{\omega}\left(\delta^{\prime} \mathbf{1}\right)+\delta \mathbf{1}^{\prime} \omega \\
& =S \omega-\lambda+\bar{\omega}\left(\delta^{\prime} \mathbf{1}\right) \mathbf{1}+\delta \\
& =\left(\lambda_{0}+\bar{\omega} \delta^{\prime} \mathbf{1}\right) \mathbf{1} .
\end{aligned}
$$

The second equality follows from the fact that $\omega_{i} \lambda_{i}=0$ for all $i$, and $\delta_{i}\left(\omega_{i}-\right.$ $\bar{\omega})=0$ for all $i$. The third equality holds because $\sum_{j} w_{j}=1$, and the last 
equality follows from (5). The fact that $\tilde{S} \omega=\left(\lambda_{0}+\bar{\omega} \delta^{\prime} \mathbf{1}\right) \mathbf{1}$ shows that $\omega$ solves the (unconstrained) variance minimization problem for covariance matrix $\tilde{S}$.

$Q E D$

Proof of Proposition 2: First, let's re-write the second constraint in the constrained MLE problem as

$$
(1-\bar{\omega}) \sum_{j} \Omega^{i, j} \leq \bar{\omega} \sum_{k \neq i} \sum_{j} \Omega^{k, j} .
$$

Let the constrained MLE be $\hat{\Omega}$. The Kuhn-Tucker conditions for the constrained MLE problem are (Morrison (1990), p. 99):

$$
\begin{aligned}
\frac{\partial l}{\partial \Omega^{i, i}} & =\frac{1}{2} \hat{\Omega}_{i, i}-\frac{1}{2} S_{i, i}=-\lambda_{i}+(1-\bar{\omega}) \delta_{i}, \text { all } i \\
\frac{\partial l}{\partial \Omega^{i, j}} & =\hat{\Omega}_{i, j}-S_{i, j}=-\left(\lambda_{i}+\lambda_{j}\right)+(1-\bar{\omega})\left(\delta_{i}+\delta_{j}\right), \text { all } i<j \\
\lambda_{i} & \geq 0, \text { and } \lambda_{i}=0 \text { if } \sum_{j} \hat{\Omega}^{i, j}>0, \\
\delta_{i} & \geq 0, \text { and } \delta_{i}=0 \text { if }(1-\bar{\omega}) \sum_{j} \Omega^{i, j}<\bar{\omega} \sum_{k \neq i} \sum_{j} \Omega^{k, j} .
\end{aligned}
$$

These conditions imply that the constrained MLE can be written as

$$
\hat{\Omega}=S-\left(\lambda \mathbf{1}^{\prime}+\mathbf{1} \lambda^{\prime}\right)+(1-\bar{\omega})\left(\delta \mathbf{1}^{\prime}+\mathbf{1} \delta^{\prime}\right)
$$

Notice that $\hat{\Omega}$ has the same form as $\tilde{S}$.

We will only prove Part 1 . The proof of Part 2 is similar.

Let $\left\{\lambda_{i}, \delta_{i}, \omega_{i}\right\}_{i=1}^{N}$ be a solution to the constrained portfolio variance minimization problem (1) - (4), given $S$, and construct $\tilde{S}$ according to (8). Then we can easily verify that $\left\{\lambda_{i},(1-\bar{\omega}) \delta_{i}\right\}_{i=1}^{N}$ and $\tilde{S}$ satisfies $(25),(26)$, and the first halfs of (27) and (28). Now we need to verify the second halfs of (27) and (28), i.e., $\sum_{j} \tilde{S}^{i, j}>0$ implies $\lambda_{i}=0$ and $(1-\bar{\omega}) \sum_{j} \tilde{S}^{i, j}<\bar{\omega} \sum_{k \neq i} \sum_{j} \tilde{S}^{k, j}$ implies $\delta_{i}=0$. By Proposition 1, $\omega$ is the unconstrained global minimum variance portfolio of $\tilde{S}$, so $\omega_{i}=\sum_{j} \tilde{S}^{i, j} / \sum_{j} \sum_{k} \tilde{S}^{j, k}$. From the second half of (6) we know that $\omega_{i}>0$ implies $\lambda_{i}=0$. This says that $\sum_{j} \tilde{S}^{i, j}>0$ implies $\lambda_{i}=0$. Likewise, the second half of (7) implies the second half of (28). QED 


\section{References}

Alexander, G. J., 1978, A Reevaluation of Alternative Portfolio Selection Models Applied to Common Stocks, Journal of Financial and Quantitative Analysis 13, 71-78.

Best, Michael J. and Robert R. Grauer, 1991, On the Sensitivity of MeanVariance-Efficient Portfolios to Changes in Asset Means: Some Analytical and Computational Results, Review of Financial Studies 4, 315-42.

Bird, Ron, and Mark Tippett, 1986, Naive Diversification and Portfolio RiskA Note, Management Science 32, 244-51.

Black, Fisher and Robert Litterman, 1992, Global Portfolio Optimization, Financial Analysts Journal, September-October, 28-43.

Bloomfield, Ted, Richard Leftwich and John Long, 1977, Portfolio Strategies and Performance, Journal of Financial Economics 5, 201-218.

Chan, Louis K.C. Jason Karceski and Josef Lakonishok, 1999, On Portfolio Optimization: Forecasting Covariances and Choosing the Risk Model, Review of Financial Studies 12, 937-74.

Cohen, G., G. Hawawini, S. Maier, R. Schwartz, and D. Whitcome, 1983, Friction in the Trading Process and the Estimation of Systematic Risk, Journal of Financial Economics 12, 263-78.

Cohen, K.J. and J.A.Pogue, 1967, An Empirical Evaluation of Alternative Portfolio Selection Models, Journal of Business 40, 166-193.

Connor, Gregory, and Robert A. Korajczyk, 1986, Performance Measurement with the Arbitrage Pricing Theory, Journal of Financial Economics, 15, 373-394.

Connor, Gregory and Robert A. Korajczyk, 1988, Risk and Return in an Equilibrium APT: Applications of a New Test Methodology, Journal of Financial Economics 21, 255-289. 
Connor, Gregory and Robert A. Korajczyk, 1993, A Test for the Number of Factors in an Approximate Factor Model, Journal of Finance 48, 1263-91.

Connor, Gregory and Robert A. Korajczyk, 1995, The Arbitrage Pricing Theory and Multifactor Models of Asset Returns, in: R. A. Jarrow, V. Maksimovic, and W.T.Ziemba (eds.), Finance, Elsevier Science.

Elton, E. J. and M.J. Gruber, 1973, Estimating the Dependence Structure of Share Prices - Implications for Portfolio Selection, Journal of Finance 28, 1203-1232.

Elton, E. J., and M. J. Gruber, 1977, Risk Reduction and Portfolio Size: An Analytical Solution, Journal of Business 50, 415-37.

Elton, E. J. M. J. Gruber and T. Ulrich, 1978, Are Betas Best? Journal of Finance 23, 1375-1384.

Evans, John L. and Stephen H. Archer, 1968, Diversification and the Reduction of Dispersion: An Empirical Analysis, Journal of Finance 21, $761-67$.

Fama, Eugene, and Kenneth French, 1992, The Cross-Section of Expected Stock Returns, Journal of Finance 47, 427-465.

Fama, Eugene and Kenneth French, 1993, Common Risk Factors in the Returns on Stocks and Bonds, Journal of Financial Economics 33, 3-56.

Frost, Peter A. and James E. Savarino, 1986, An Empirical Bayes Approach to Efficient Portfolio Selection, Journal of Financial and Quantitative Analysis 21, 293-305.

Frost, Peter A. and James E. Savarino, 1988, For Better Performance: Constrain Portfolio Weights, Journal of Portfolio Management 15, No. 1, 29-34.

Green, Richard C. and Burton Hollifield, 1992, When Will Mean-Variance 
Efficient Portfolios be Well Diversified? Journal of Finance 47, 17851809.

James, W. and C. Stein, 1961, Estimation with Quadratic Loss. In Proc. Fourth Berkeley Symp. Math. Statist. Probab., 1, 361-79. University of California Press, Berkeley, CA.

Jobson, J.D. and B. Korkie, 1980, Estimation for Markowitz Efficient Portfolios, Journal of the American Statistical Association 75, 544-54.

Jobson, J.D. and B. Korkie, 1981, Putting Markowitz Theory to Work, Journal of Portfolio Management, Summer, 70-74.

Johnson, K. H. and D. S. Shannon, 1974, A Note on Diversification and the Reduction of Dispersion, Journal of Financial Economics 1, 365-72.

Jorion, Philippe, 1985, International Portfolio Diversification with Estimation Risk, Journal of Business, 58, 3, 259-278.

Jorion, Philippe, 1986, Bayes-Stein Estimation for Portfolio Analysis, Journal of Financial and Quantitative Analysis, 21, 3, 279-292.

Jorion, Philippe, 1991, Bayesian and CAPM estimators of the means: Implications for portfolio selection, Journal of Banking and Finance, 15, 717-727.

Ledoit, Olivier, 1996, A Well-Conditioned Estimator for Large Dimensional Covariance Matrices, Unpublished, UCLA.

Ledoit, Olivier, 1999, Improved Estimation of the Covariance Matrix of Stock Returns with an Application to Portfolio Selection, Unpublished, UCLA.

Liu, Ludan, 2001, Portfolio Constraints and Shrinkage Estimation, Unpublishes, Carroll School of Management, Boston College.

MacKinlay, A. Craig and Lubos Pastor, 1999, Asset Pricing Models: Implications for Expected Returns and Portfolio Selection, Unpublished, The Wharton School, University of Pennsylvania. 
Markowitz, H. M., 1952, Portfolio Selection, Journal of Finance 7, 77-91.

Markowitz, H. M., 1959, Portfolio Selection: Efficient Diversification of Investments, Yale University Press, New Haven, CT.

Michaud, Richard O. 1989, The Markowitz Optimization Enigma: Is 'Optimized' Optimal? Financial Analysts Journal, January-February, 31-42.

Morrison, Donald F., 1990, Multivariate Statistical Methods (3rd ed.), McGrawHill.

Newy, Whitney K, and Kenneth D. West, 1987, A Simple, Positive SemiDefinite, Heteroskedasticity and Autocorrelation Consistent Covariance Matrix, Econometrica 55, 703-708.

Scholes, M. and J. Williams, 1977, Estimating Betas from Nonsynchronous Data, Journal of Financial Economics 5, 309-28.

Shanken, Jay, 1987, Nonsynchronous Data and the Covariance-Factor Structure of Returns, Journal of Finance 42, 221-31.

Sharpe, William, 1963, A Simplified Model for Portfolio Analysis Management Science 9, 277-293.

Statman, Meir, 1987, How Many Stocks Make a Diversified Portfolio? Journal of Financial and Quantitative Analysis 22, 353-63.

Tole, Thomas M., 1982, You Can’t Diversify without Diversifying, Journal of Portfolio Management, Winter, 5-11.

Wallingford, Buckner, 1967, A Survey and Comparison of Portfolio Selection Models, Journal of Financial and Quantitative Analysis 2, 85-106. 


\section{Table 1. The effect of portfolio weight constraints}

This table shows the effects of nonnegativity constraints and upper bound on portfolio weights. The covariance matrix $\mathrm{S}$ is the sample covariance matrix of monthly returns of a random sample of 30 stocks from 1988-1997 (120 months). $\lambda$ and $\delta$ are the vectors of Lagrange multipliers on the nonnegativity constraint and the upper bound, respectively. The upper bound is set at $10 \%$. The numbers below the correlation coefficients (and in parenthesis) are significant levels. The numbers below the regression coefficients are standard errors.

\begin{tabular}{|c|c|c|c|c|}
\hline \multicolumn{5}{|c|}{$\begin{array}{l}\text { Panel A: Summary statistics of the row sums of S, unconstrained portfolio weights, and } \\
\text { the Lagrange multipliers }\end{array}$} \\
\hline Variable & Mean & Std Dev & Minimum & Maximum \\
\hline Row sums of $\mathrm{S}$ & 0.0358 & 0.0169 & 0.0106 & 0.0695 \\
\hline Unconstrained portfolio weights & 0.0333 & 0.0926 & -0.1251 & 0.3569 \\
\hline$\lambda_{i}^{\prime} s$ & 0.000063 & 0.000092 & 0 & 0.000316 \\
\hline$\delta_{i}^{\prime} s$ & 0.000016 & 0.000045 & 0 & 0.000169 \\
\hline
\end{tabular}

Panel B: Correlations among the row sums of S, unconstrained portfolio weights, and the Lagrange multipliers

Row sum's of S

Unconstrained portfolio weights

$\lambda_{i}$ s
Unconstrained portfolio weights

-0.496
$(0.0053)$

$$
\lambda_{i}{ }^{\prime} s
$$$$
\delta_{\mathrm{i}} \text { 's }
$$

\begin{tabular}{rr}
0.713 & -0.425 \\
$(0.0001)$ & $(0.0191)$ \\
-0.494 & 0.808 \\
$(0.0055)$ & $(0.0001)$ \\
-0.255 \\
& $(0.173)$ \\
\hline
\end{tabular}

Panel C: Summary statistics for the (distinct) individual elements of $\mathrm{S}$, and the adjustments to the individual elements due to the portfolio weight constraints

\begin{tabular}{lrrrr}
\hline Variable & Mean & Std Dev & Minimum & Maximum \\
\hline $\mathrm{S}_{\mathrm{ij}}$ & 0.00139 & 0.00216 & -0.00186 & 0.019 \\
$\lambda_{\mathrm{i}}+\lambda_{\mathrm{j}}$ & 0.000127 & 0.00013 & 0 & 0.000632 \\
$\delta_{\mathrm{i}}+\delta_{\mathrm{j}}$ & 0.000032 & 0.000063 & 0 & 0.000338 \\
\hline
\end{tabular}

Panel D: Correlations among the (distinct) individual elements of S and the adjustments to the individual elements due to the portfolio weight constraints

\begin{tabular}{lrr}
\hline & $\lambda_{\mathrm{i}}+\lambda_{\mathrm{j}}$ & $\delta_{\mathrm{i}}+\delta_{\mathrm{j}}$ \\
\cline { 2 - 3 } $\mathrm{S}_{\mathrm{ij}}+\lambda_{\mathrm{j}}$ & 0.296 & -0.184 \\
& $(0.0001)$ & $(0.0001)$ \\
& -0.255 \\
& & $(0.0001)$ \\
\hline
\end{tabular}

Panel E: Regression of the adjustments on the (distinct) individual elements of $\mathrm{S}$

\begin{tabular}{lrrrr}
\hline Dependent Variable & Intercept & $\mathrm{S}_{\mathrm{ij}}$ & No. of obs. & Adj. $\mathrm{R}^{2}$ \\
\hline$\lambda_{\mathrm{i}}+\lambda_{\mathrm{j}}$ & $1.02 \mathrm{E}-4$ & 0.0177 & 465 & 0.085 \\
$\delta_{\mathrm{i}}+\delta_{\mathrm{j}}$ & $(6.84 \mathrm{E}-6)$ & $(0.00266)$ & & \\
& $3.94 \mathrm{E}-5$ & -0.00539 & 465 & 0.032 \\
\hline
\end{tabular}




\section{Table 2. Effects of imposing nonnegativity constraints and the single-factor constraint}

This table shows the percentage reduction in the ex post standard deviations of the global minimum variance portfolios, when the nonnegativity and/or single factor model constraints are imposed, relative the ex post variances of the global minimum variance portfolios constructed from the sample covariance matrix and the equal-weighted portfolio. $\mathrm{N}$ is the number of assets; $\mathrm{T}$ is the sample size used to estimate the covariance matrix. $\sigma_{\beta}$ is the cross-sectional standard deviation in the stocks' betas with respect to the first factor. The true stock returns have a two factor structure, and the covariance structure roughly matches that of the NYSE when $\sigma_{\beta}$ is 0.4 . Refer to the text for details of the setup of the simulation. When $\sigma_{\beta}$ is 0 , the nonnegativity constraint is correct in population; when $\sigma_{\beta}$ is 0.4 , the nonnegativity constraint is violated roughly to the same extent as using the NYSE stocks; and when $\sigma_{\beta}$ is 0.2 , the nonnegativity constraint is more severely violated than when $\sigma_{\beta}$ is 0.4 . The rows with $\mathrm{T}=$ Infinity are the population results.

\begin{tabular}{|c|c|c|c|c|c|c|c|}
\hline \multirow[t]{3}{*}{$\mathrm{N}$} & \multirow[t]{3}{*}{$\mathrm{T}$} & \multicolumn{6}{|c|}{$\begin{array}{l}\text { Percentage reduction in ex post standard deviation of the optimal portfolios } \\
\text { from imposing the constraints }\end{array}$} \\
\hline & & \multicolumn{3}{|c|}{$\begin{array}{l}\text { Relative to the portfolio constructed } \\
\text { from the sample covariance matrix }\end{array}$} & \multicolumn{3}{|c|}{$\begin{array}{l}\text { Relative to the equal-weighted } \\
\text { portfolio }\end{array}$} \\
\hline & & $\begin{array}{r}\text { Single- } \\
\text { factor } \\
\text { model } \\
\end{array}$ & $\begin{array}{r}\text { Nonnegativity } \\
\text { constraint }\end{array}$ & Both & $\begin{array}{r}\text { Single- } \\
\text { factor } \\
\text { model }\end{array}$ & $\begin{array}{r}\text { Nonnegativity } \\
\text { constraint }\end{array}$ & Both \\
\hline \multicolumn{8}{|c|}{ Panel A: $\sigma_{\beta}=0$} \\
\hline 30 & 60 & 21.6 & 25.2 & 26.0 & -12.2 & -7.0 & -6.0 \\
\hline 30 & 360 & 0.8 & 1.2 & 1.4 & -1.7 & -1.3 & -1.0 \\
\hline 30 & Infinity & 0.0 & 0.0 & 0.0 & 1.1 & 1.1 & 1.1 \\
\hline 300 & 60 & na & na & na & -8.1 & -11.6 & -6.9 \\
\hline 300 & 360 & 54.6 & 58.8 & 59.1 & -14.6 & -3.9 & -3.2 \\
\hline 300 & Infinity & 0.0 & 0.0 & 0.0 & 0.2 & 0.2 & 0.2 \\
\hline \multicolumn{8}{|c|}{ Panel B: $\sigma_{\beta}=0.2$} \\
\hline 30 & 60 & 20.7 & 16.2 & 18.7 & 8.4 & 3.3 & 6.1 \\
\hline 30 & 360 & 1.7 & -3.2 & -2.6 & 14.8 & 10.6 & 11.1 \\
\hline 30 & Infinity & -0.3 & -5.6 & -5.8 & 16.8 & 12.4 & 12.3 \\
\hline 300 & 60 & na & na & na & 45.9 & 18.1 & 23.2 \\
\hline 300 & 360 & 56.2 & 26.1 & 26.5 & 60.3 & 33.0 & 33.3 \\
\hline 300 & Infinity & -5.0 & -80.7 & -81.3 & 62.8 & 35.9 & 35.7 \\
\hline \multicolumn{8}{|c|}{ Panel C: $\sigma_{\beta}=0.4$} \\
\hline 30 & 60 & 27.2 & 16.8 & 21.2 & 38.9 & 30.2 & 33.9 \\
\hline 30 & 360 & 2.3 & -7.6 & -7.1 & 42.6 & 36.8 & 37.0 \\
\hline 30 & Infinity & -0.1 & -10.0 & -10.0 & 43.3 & 37.7 & 37.7 \\
\hline 300 & 60 & na & na & na & 72.9 & 50.8 & 57.6 \\
\hline 300 & 360 & 59.2 & 25.0 & 27.2 & 79.3 & 61.9 & 63.0 \\
\hline 300 & Infinity & -0.8 & -79.4 & -80.7 & 79.9 & 64.2 & 64.0 \\
\hline
\end{tabular}


Table 3. Cutoff points for the sample sizes when the nonnegativity constraints start to hurt.

The true return covariance has a two factor structure. Across stocks, the betas with respect to the first factor is normally distributed with mean one and standard deviation $\delta_{\beta}$. The number of stocks is $\mathrm{N}$, and the sample size is $\mathrm{T}$. We estimate the covariance matrix for the stocks using simulated returns of sample size $T$ using the sample covariance matrix. Then we form the nonnegativity constrained and unconstrained global minimum variance portfolios using the estimated covariance matrix, and calculate the ex post variances of these portfolios. For each $\left(\delta_{\beta}, N\right)$ pair, we report the cutoff point for T such that when the sample size is greater than or equal to the cutoff point, the nonnegativity constraint will hurt. For more details of the simulation specification, please refer to the text.

\begin{tabular}{cccc}
\hline $\mathrm{N}$ & $\delta_{\beta}$ & & \\
\cline { 2 - 4 } & 0.2 & 0.3 & 0.4 \\
\hline 30 & $\mathrm{na}^{\mathrm{l}}$ & 120 & 120 \\
60 & 150 & 150 & 150 \\
120 & 270 & 270 & 330 \\
180 & 330 & 270 & 330 \\
240 & 390 & 390 & 450 \\
300 & 450 & 450 & 450 \\
\hline
\end{tabular}

${ }^{1}$ : Such cutoff point is not found in that set of simulation. 


\section{Table 4. Short interests in the global minimum variance portfolios}

The true return covariance has a two factor structure. Across stocks, the betas with respect to the first factor is normally distributed with mean one and standard deviation $\delta_{\beta}$, the betas with respect to the second factor is normally distributed with mean zero and standard deviation 0.2. The two factor returns have unit variances and zero covariance. The stocks' residual variances are constant over time, but follow a Lognormal $(0.8,0.7)$ distribution. The number of stocks is N. For each $\left(\delta_{\beta}, N\right)$ pair, we draw the betas and the residual variances according to the above-mentioned distributional assumptions, and then calculate the true stock return covariance matrix. We then calculate the unconstrained global minimum variance portfolio and its short interest (i.e., the sum of the negative portfolio weights). This procedure is repeated ten times and the averages are reported below. The short interests give the extent to which the nonnegativity constraint is wrong.

\begin{tabular}{|c|c|c|c|c|}
\hline \multirow[t]{2}{*}{$\underline{N}$} & \multicolumn{3}{|c|}{$\delta_{\beta}$} & \multirow[b]{2}{*}{0.4} \\
\hline & 0.1 & 0.2 & 0.3 & \\
\hline 30 & -0.181 & -0.401 & -0.391 & -0.350 \\
\hline 60 & -0.554 & -0.706 & -0.584 & -0.468 \\
\hline 120 & -1.188 & -1.012 & -0.737 & -0.515 \\
\hline 180 & -1.578 & -1.161 & -0.828 & -0.555 \\
\hline 240 & -1.845 & -1.250 & -0.816 & -0.534 \\
\hline 300 & -2.042 & -1.308 & -0.831 & -0.526 \\
\hline
\end{tabular}


Table 5. Ex post mean, standard deviation, and other characteristics of the global minimum variance portfolios

At the end of April each year from 1968-1997, the covariance matrix of a random sample of 500 stocks is estimated according to variance estimators. We use these covariance matrix estimates to construct the global minimum variance portfolios, both constrained and unconstrained. We hold the portfolios for the next 12 months and their monthly returns are recorded. The ex post means, standard deviations, and other characteristics of these portfolios are reported. The $\mathrm{C}$ after an estimator indicates the nonnegativity constrained portfolios, and $\mathrm{D}$ after an estimator indicates the portfolio with both the nonnegativity constraint and the upper bound of $2 \%$. For the equal-weighted and valueweighted portfolios of 25 stocks, the 25 stocks are randomly selected from the 500 ones. Means and standard deviations are in percentage per year, maximum weight, minimum weight, and short interest are in percentage.

\begin{tabular}{|c|c|c|c|c|c|c|}
\hline Covariance matrix estimator & Mean & $\begin{array}{l}\text { Std } \\
\text { Dev }\end{array}$ & $\begin{array}{l}\text { Max. } \\
\text { weight }\end{array}$ & $\begin{array}{l}\text { Min. } \\
\text { weight }\end{array}$ & $\begin{array}{l}\text { Short } \\
\text { interest }\end{array}$ & $\begin{array}{l}\text { No. of } \\
\text { positive } \\
\text { weights }\end{array}$ \\
\hline Monthly sample covariance matrix, $\mathrm{C}$ & 13.55 & 12.43 & 18.4 & 0 & 0 & 24.1 \\
\hline Monthly sample covariance matrix, D & 13.55 & 12.85 & 2.0 & 0 & 0 & 59.5 \\
\hline One-factor model & 13.99 & 11.69 & 3.8 & -0.9 & -50.7 & 268.8 \\
\hline One-factor model, $\mathrm{C}$ & 12.68 & 12.62 & 11.3 & 0 & 0 & 39.3 \\
\hline One-factor model, D & 13.51 & 12.50 & 2.0 & 0 & 0 & 63.1 \\
\hline Ledoit & 13.09 & 10.76 & 4.9 & -1.7 & -80.6 & 283.2 \\
\hline Ledoit, C & 12.79 & 12.29 & 13.4 & 0 & 0 & 39.7 \\
\hline Ledoit, D & 13.49 & 12.43 & 2.0 & 0 & 0 & 65.4 \\
\hline Fama-French 3 factor model & 13.04 & 11.35 & 4.2 & -1.4 & -63.5 & 284.1 \\
\hline Fama-French 3 factor model, C & 12.65 & 12.38 & 12.2 & 0 & 0 & 40.1 \\
\hline Fama-French 3 factor model, D & 13.34 & 12.53 & 2.0 & 0 & 0 & 64.0 \\
\hline Daily sample covariance matrix & 14.06 & 10.64 & 6.3 & -2.5 & -122.4 & 270.7 \\
\hline Daily sample covariance matrix, $\mathrm{C}$ & 13.95 & 12.34 & 8.8 & 0 & 0 & 64.7 \\
\hline Daily sample covariance matrix, D & 14.22 & 12.28 & 2.0 & 0 & 0 & 81.1 \\
\hline $\begin{array}{l}\text { Daily sample covariance matrix of } \\
\text { CHMSW, C }\end{array}$ & 13.89 & 12.31 & 9.1 & 0 & 0 & 62.5 \\
\hline $\begin{array}{l}\text { Daily sample covariance matrix of } \\
\text { CHMSW, D }\end{array}$ & 14.18 & 12.25 & 2.0 & 0 & 0 & 80.1 \\
\hline Daily sample covariance matrix (New) & 13.94 & 10.60 & 6.3 & -2.6 & -128.5 & 269.5 \\
\hline Daily sample covariance matrix (New), C & 13.81 & 12.26 & 9.0 & 0 & 0 & 62.9 \\
\hline Daily sample covariance matrix (New), D & 14.12 & 12.21 & 2.0 & 0 & 0 & 79.5 \\
\hline Daily Fama-French 3-factor & 13.07 & 11.25 & 4 & -1.4 & -66.9 & 276.8 \\
\hline Daily Fama-French 3-factor, C & 13.03 & 12.03 & 12.7 & 0 & 0 & 40.7 \\
\hline Daily Fama-French 3-factor, D & 13.09 & 12.15 & 2 & 0 & 0 & 63.6 \\
\hline Equal-weighted portfolio of the 500 stocks & 14.52 & 17.48 & 0.2 & 0 & 0 & 500 \\
\hline Value-weighted portfolio of the 500 stocks & 13.39 & 15.60 & 7.3 & 0 & 0 & 500 \\
\hline Equal-weighted portfolio of 25 stocks & 15.16 & 17.78 & 4 & 0 & 0 & 25 \\
\hline Value-weighted portfolio of 25 stocks & 14.25 & 17.79 & 30 & 0 & 0 & 25 \\
\hline
\end{tabular}


Table 6. Ex post mean, standard deviation, and other characteristics of the minimum tracking error variance portfolios

At the end of April each year from 1968-1997, the covariance matrix of a random sample of 500 stocks is estimated according to variance estimators. We use these covariance matrix estimates to construct the minimum tracking error variance portfolios, both constrained and unconstrained. The target is the S\&P 500 returns. We hold the portfolios for the next 12 months and their monthly tracking errors are recorded. The ex post means and standard deviations of the tracking errors and some characteristics of the tracking portfolios are reported. The $\mathrm{C}$ after an estimator indicates the nonnegativity constrained portfolios, and D after an estimator indicates the portfolio with both the nonnegativity constraint and the upper bound of $2 \%$. For the equal-weighted and value-weighted portfolios of 25 stocks, the 25 stocks are randomly selected from the 500 ones. Means and standard deviations are in percentage per year, maximum weight, minimum weight, and short interest are in percentage.

\begin{tabular}{|c|c|c|c|c|c|c|}
\hline Covariance matrix estimator & Mean & $\begin{array}{l}\text { Std } \\
\text { Dev }\end{array}$ & $\begin{array}{l}\text { Max. } \\
\text { weight }\end{array}$ & $\begin{array}{l}\text { Min. } \\
\text { weight }\end{array}$ & $\begin{array}{l}\text { Short } \\
\text { interest }\end{array}$ & $\begin{array}{l}\text { No. of } \\
\text { positive } \\
\text { weights }\end{array}$ \\
\hline Monthly sample covariance matrix, $\mathrm{C}$ & 4.28 & 3.36 & 2.4 & 0 & 0 & 200 \\
\hline Monthly sample covariance matrix, D & 4.23 & 3.40 & 1.8 & 0 & 0 & 194 \\
\hline One-factor model & 4.91 & 5.04 & 0.9 & 0 & 0 & 499 \\
\hline One-factor model, $\mathrm{C}$ & 4.91 & 5.04 & 0.9 & 0 & 0 & 499 \\
\hline One-factor model, D & 4.91 & 5.04 & 0.9 & 0 & 0 & 499 \\
\hline Ledoit & 4.18 & 3.48 & 1.8 & -0.3 & -8.7 & 391 \\
\hline Ledoit, C & 4.21 & 3.34 & 2.2 & 0 & 0 & 314 \\
\hline Ledoit, D & 4.27 & 3.36 & 1.8 & 0 & 0 & 312 \\
\hline Fama-French 3 factor model & 3.76 & 4.41 & 1.4 & -0.2 & -3.3 & 433.1 \\
\hline Fama-French 3 factor model, C & 3.71 & 4.39 & 1.5 & 0 & 0 & 394.6 \\
\hline Fama-French 3 factor model, D & 3.71 & 4.39 & 1.5 & 0 & 0 & 394.5 \\
\hline Daily sample covariance matrix & 3.69 & 2.94 & 5.3 & -1 & -36.6 & 322.4 \\
\hline Daily sample covariance matrix, $\mathrm{C}$ & 3.93 & 2.78 & 5.4 & 0 & 0 & 231.3 \\
\hline Daily sample covariance matrix, D & 4.17 & 2.96 & 2 & 0 & 0 & 227.6 \\
\hline $\begin{array}{l}\text { Daily sample covariance matrix of } \\
\text { CHMSW, C }\end{array}$ & 3.92 & 2.75 & 5.4 & 0 & 0 & 231.4 \\
\hline $\begin{array}{l}\text { Daily sample covariance matrix of } \\
\text { CHMSW, D }\end{array}$ & 4.17 & 2.92 & 2 & 0 & 0 & 226.3 \\
\hline Daily sample covariance matrix (New) & 3.72 & 2.92 & 5.3 & -1 & -36.8 & 322.2 \\
\hline Daily sample covariance matrix (New), C & 3.93 & 2.73 & 5.4 & 0 & 0 & 228 \\
\hline Daily sample covariance matrix (New), D & 4.17 & 2.89 & 2 & 0 & 0 & 224.2 \\
\hline Daily Fama-French 3-factor & 3.82 & 4.50 & 1.3 & -0.2 & -3.7 & 427.8 \\
\hline Daily Fama-French 3-factor, C & 3.76 & 4.50 & 1.5 & 0 & 0 & 384.1 \\
\hline Daily Fama-French 3-factor, D & 3.77 & 4.51 & 1.4 & 0 & 0 & 383.8 \\
\hline Equal-weighted portfolio of the 500 stocks & 4.92 & 6.58 & 0.2 & 0 & 0 & 500 \\
\hline Value-weighted portfolio of the 500 stocks & 3.6 & 2.37 & 6.7 & 0 & 0 & 500 \\
\hline Equal-weighted portfolio of 25 stocks & 4.66 & 8.62 & 4 & 0 & 0 & 25 \\
\hline Value-weighted portfolio of 25 stocks & 4.13 & 8.24 & 29.7 & 0 & 0 & 25 \\
\hline
\end{tabular}




\section{Table 7. t-tests of equal mean returns and equal mean squared returns}

This table reports the t-tests of equal mean returns and equal mean squared returns of the minimum variance and minimum tracking error variance portfolios. For each such portfolio, we test whether its mean returns and mean squared returns are statistically different from those of the nonnegativity constrained portfolio constructed from the sample covariance matrix of monthly returns.

\begin{tabular}{|c|c|c|c|c|}
\hline \multirow[t]{2}{*}{ Covariance matrix estimator } & \multicolumn{2}{|c|}{$\begin{array}{l}\text { Minimum variance } \\
\text { portfolio }\end{array}$} & \multicolumn{2}{|c|}{$\begin{array}{l}\text { Minimum tracking error } \\
\text { portfolio }\end{array}$} \\
\hline & $\begin{array}{l}\text { Equality } \\
\text { in mean } \\
\text { return }\end{array}$ & $\begin{array}{c}\text { Equality in } \\
\text { mean squared } \\
\text { return }\end{array}$ & $\begin{array}{l}\text { Equality } \\
\text { in mean } \\
\text { return }\end{array}$ & $\begin{array}{c}\text { Equality in } \\
\text { mean squared } \\
\text { return }\end{array}$ \\
\hline Monthly sample covariance matrix, D & -0.01 & 1.36 & -0.53 & 1.49 \\
\hline One-factor model & 0.26 & -1.16 & 1.06 & 5.35 \\
\hline One-factor model, $\mathrm{C}$ & -0.76 & 0.19 & 1.06 & 5.34 \\
\hline One-factor model, D & -0.05 & 0.15 & 1.06 & 5.34 \\
\hline Ledoit & -0.40 & -2.99 & -0.32 & 1.06 \\
\hline Ledoit, $\mathrm{C}$ & -1.05 & -0.60 & -0.30 & -0.32 \\
\hline Ledoit, D & -0.07 & -0.02 & -0.03 & 0.08 \\
\hline Fama-French 3 factor model & -0.38 & -2.34 & -1.02 & 4.46 \\
\hline Fama-French 3 factor model, C & -1.08 & -0.29 & -1.15 & 4.42 \\
\hline Fama-French 3 factor model, D & -0.22 & 0.23 & -1.15 & 4.41 \\
\hline Daily sample covariance matrix & 0.38 & -3.86 & -1.30 & -3.29 \\
\hline Daily sample covariance matrix, $\mathrm{C}$ & 0.35 & -0.11 & -0.87 & -4.43 \\
\hline Daily sample covariance matrix, D & 0.65 & -0.22 & -0.28 & -3.26 \\
\hline $\begin{array}{l}\text { Daily sample covariance matrix of } \\
\text { CHMSW, C }\end{array}$ & 0.29 & -0.20 & -0.89 & -4.82 \\
\hline $\begin{array}{l}\text { Daily sample covariance matrix of } \\
\text { CHMSW, D }\end{array}$ & 0.62 & -0.31 & -0.29 & -3.72 \\
\hline Daily sample covariance matrix (New) & 0.29 & -3.82 & -1.24 & -3.40 \\
\hline Daily sample covariance matrix (New), C & 0.23 & -0.35 & -0.87 & -5.09 \\
\hline Daily sample covariance matrix (New), D & 0.57 & -0.44 & -0.29 & -4.11 \\
\hline Daily Fama-French 3-factor & -0.31 & -2.41 & -0.82 & 4.28 \\
\hline Daily Fama-French 3-factor, C & -0.51 & -0.95 & -0.95 & 4.25 \\
\hline Daily Fama-French 3-factor, D & -0.44 & -0.78 & -0.93 & 4.26 \\
\hline Equal-weighted portfolio of the 500 stocks & 0.54 & 6.79 & 0.73 & 6.76 \\
\hline Value-weighted portfolio of the 500 stocks & -0.10 & 5.25 & -1.48 & -7.33 \\
\hline Equal-weighted portfolio of 25 stocks & 0.82 & 6.94 & 0.29 & 9.48 \\
\hline Value-weighted portfolio of 25 stocks & 0.32 & 7.07 & -0.10 & 9.88 \\
\hline
\end{tabular}


The assets are the Fama-French 25 size-beta sorted portfolios.

The covariance matrix is estimated at the end of April, using monthly

returns of the previous 5 year. The tangency portfolios and the global minimum variance portfolios are then formed

and held for one year. This procedure is repeated from 1968 to 1998.

The table reports characteristics of ex post excess returns of these portfolios.

Both unconstrained and nonnegativity constrained portfolios are considered.

Means and standard deviations of returns are monthly ones. Sharpe ratios are annualized.

\begin{tabular}{|c|c|c|c|c|c|c|c|c|c|c|}
\hline Portfolios & $\begin{array}{c}\text { in-sample } \\
\text { mean }\end{array}$ & $\begin{array}{c}\text { in-sample } \\
\text { std dev }\end{array}$ & $\begin{array}{c}\text { Sharpe } \\
\text { Ratio }\end{array}$ & Mean & Std Dev & Minimum & Maximum & $\begin{array}{c}\text { Sharpe } \\
\text { Ratio }\end{array}$ & $\begin{array}{l}\text { Average } \\
\text { total short } \\
\text { position }\end{array}$ & $\begin{array}{c}\text { Average No. } \\
\text { of assets } \\
\text { held long }\end{array}$ \\
\hline
\end{tabular}

\section{Panel A: Tangency portfolios}

Sample cov matrix, constrained

Sample cov matrix, unconstrained

One-factor model, constrained

One-factor model, unconstrained

FF 3-factor model, constrained

FF 3-factor model, unconstrained

Ledoit estimator, constrained

Ledoit estimator, unconstrained

Equally-weighted portfolio

1.01
-2.96
0.97
6.27
1.02
18.03
1.01
-208.78

$\begin{array}{rr}5.34 & 0.66 \\ 14.01 & -0.73 \\ 5.19 & 0.65 \\ 10.57 & 2.06 \\ 5.35 & 0.66 \\ 42.63 & 1.46 \\ 5.31 & 0.66 \\ 409.80 & -1.76\end{array}$

0.72
0.80
0.67
4.87
0.71
-0.33
0.71
-66.40

5.59
43.94
5.64
46.31
5.60
83.32
5.58
1851.19

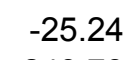

$-243.78$

$-26.19$

$-160.16$

$-25.24$

$-715.70$

$-25.24$

0.62

5.15

$-19304.37$

$-26.56$

sample cov matrix, unconstrained

one-factor model, constrained

one-factor model, unconstrained

FF 3-factor model, constrained

FF 3-factor model, unconstrained

Ledoit Estimator, constrained

Ledoit Estimator, unconstrained

Equally-weighted portfolio
4.33

4.60

4.60

0.58
0.80
0.58
0.69
0.58
0.85
0.58
0.71

\begin{tabular}{lll}
0.46 & 0.51 & 3.75 \\
0.60 & 0.75 & 2.15 \\
0.45 & 0.54 & 3.73 \\
0.49 & 0.65 & 2.43 \\
0.46 & 0.51 & 3.77 \\
0.72 & 0.68 & 2.48 \\
0.46 & 0.52 & 3.76 \\
0.61 & 0.73 & 2.68 \\
& 0.62 & 5.15 \\
\hline
\end{tabular}

4.82
4.36

4.36
4.13

4.34
4.04

\begin{tabular}{ll}
-24.42 & 16.78 \\
-21.95 & 17.44 \\
-26.34 & 17.95 \\
-19.75 & 20.16 \\
-24.66 & 17.79 \\
-20.93 & 18.27 \\
-24.52 & 17.00 \\
-20.85 & 16.29 \\
-26.56 & 22.54 \\
\hline
\end{tabular}

22.54

$\begin{array}{rrrr}25.74 & 0.45 & 0.00 & 2.29 \\ 193.46 & 0.06 & -36.69 & 12.68 \\ 26.78 & 0.41 & 0.00 & 4.16 \\ 500.39 & 0.36 & -9.23 & 12.94 \\ 25.74 & 0.44 & 0.00 & 2.32 \\ 533.18 & -0.01 & -51.97 & 12.81 \\ 25.74 & 0.44 & 0.00 & 2.55 \\ 15046.03 & -0.12 & -611.30 & 13.16 \\ 22.54 & 0.41 & 0.00 & 25.00\end{array}$

\begin{tabular}{rrr}
0.46 & 0.00 & 3.58 \\
0.60 & -4.82 & 13.00 \\
0.45 & 0.00 & 4.55 \\
0.49 & -1.48 & 12.35 \\
0.46 & 0.00 & 3.42 \\
0.72 & -2.52 & 13.03 \\
0.46 & 0.00 & 4.06 \\
0.61 & -2.23 & 13.39 \\
0.41 & 0.00 & 25.00 \\
\hline
\end{tabular}

